\title{
On the Existence of Feigenbaum's Fixed Point
}

\author{
M. Campanino* and H. Epstein \\ Institut des Hautes Etudes Scientifiques, F-91440 Bures-sur-Yvette, France
}

\begin{abstract}
We give a proof of the existence of a $\mathscr{C}^{2}$, even solution of Feigenbaum's functional equation

$$
g(x)=-\lambda_{0}^{-1} g\left(g\left(-\lambda_{0} x\right)\right), g(0)=1,
$$

where $g$ is a map of $[-1,1]$ into itself. It extends to a real analytic function over $\mathbb{R}$.
\end{abstract}

\section{Introduction}

In this paper we give the details of the work described in [1] by the same authors and Ruelle. While the latter is not responsible for possible mistakes in the present paper, he is, of course, a coauthor of the rest. Our purpose is to prove the existence of a $\mathscr{C}^{2}$ solution of Feigenbaum's functional equation.

$$
\left\{\begin{array}{l}
g(x)=-\frac{1}{\lambda_{0}} g\left(g\left(-\lambda_{0} x\right)\right), \\
g(0)=1
\end{array}\right.
$$

where $g$ is a map of the interval $[-1,1]$ into itself. This equation and its solution play an important role in the theory, initiated by Feigenbaum [7] concerning universal properties of one-parameter families of maps of the interval. Excellent introductions to this theory can be found in [7-9] and particularly in several works of Collet, Eckmann, Koch, and Lanford [2-5], so that we shall give no further details. It is important to note that, to each $\varepsilon>0$, corresponds the problem of finding a solution of (1) behaving, for small $|x|$, like $1-\operatorname{const}|x|^{1+\varepsilon}$ : for sufficiently small $\varepsilon$ the problem has been fully and satisfactorily solved by Collet et al. [5], who show that there is an $\varepsilon$-dependent solution $g_{\varepsilon}=f_{\varepsilon}\left(|x|^{1+\varepsilon}\right), f_{\varepsilon}$ analytic.

* Permanent address: Istituto Matematico “G. Castelnuovo”, Università degli studi di Roma, Piazzale A. Moro, I-00100 Roma, Italy 
The problem $\varepsilon=1$, (this is implied by requiring $g$ to be $\mathscr{C}^{2}$ and $g^{\prime \prime}(0)<0$ ), is of particular importance. Lanford has given a solution (see [11]) making essential use of computers. In view of the interest of the subject, it seems to be worthwhile to present the alternative solution described in this paper.

\section{The Problem to be Solved}

We look for a $\mathscr{C}^{2}$, even solution of (1). From (1) it follows that

$$
\lambda_{0}=-g(1) \text {. }
$$

Let us define, for any $\mathscr{C}^{2}$ real function $\phi$ on $[-1,1]$, and any real $\lambda$ with $0<|\lambda| \leqq 1$,

$$
m(\phi, \lambda)=M_{-\lambda}^{-1} \circ \phi \circ \phi \circ M_{-\lambda},
$$

with

$$
M_{-\lambda} x=-\lambda x
$$

i.e.

$$
(m(\phi, \lambda))(x)=-\frac{1}{\lambda} \phi(\phi(-\lambda x))
$$

For any real $\mu \in[-1,1]$,

$$
M_{\mu}^{-1} m(\phi, \lambda) M_{\mu}=m\left(M_{\mu}^{-1} \phi M_{\mu}, \lambda\right) .
$$

If $\phi$ is even, so is $m(\phi, \lambda)$. Suppose that $\phi$ is an even, concave fixed point of $m(\cdot, \lambda)$ with a maximum at $0, \phi(0)=\mu, 0<\mu<1$. Then $M_{\mu}^{-1} \phi M_{\mu}$ takes the value 1 at 0 . However the condition $\phi(0)=1$ is not preserved by the map $m(\cdot, \lambda)$. This can be remedied by several methods, in particular by using the C.E.L. map [4],

$$
J(\phi)=m(\phi,-\phi(1)) .
$$

We shall instead deal with $\omega(\phi, \lambda)$ defined by:

$$
(\omega(\phi, \lambda))(x)=-\frac{1}{\lambda} \phi(\phi(-\lambda x))+\frac{\phi(1)}{\lambda}+1,
$$

(where $0<\lambda<1$ ). In fact we concentrate on values of $\lambda$ in a small interval $I$ around $\lambda=0.4$, (as suggested by [7]) and find, for every $\lambda$ in $I$, a solution $\chi_{\lambda}$ of the equations

$$
\omega\left(\chi_{\lambda}, \lambda\right)=\chi_{\lambda}, \quad \chi_{\lambda}(0)=1 .
$$

We shall prove that $\chi_{\lambda}$ depends continuously on $\lambda$, and that $\chi_{2}(1)+\lambda$ takes opposite signs at the ends of $I$, hence the existence of $\lambda_{0} \in I$ such that $\chi_{\lambda_{0}}=g$ satisfies (1). Note that every solution of (1), (even or not) satisfies $g(1)+\lambda_{0}=0$ and is thus a solution of (7) with $\lambda=\lambda_{0}$. It will be seen [Eq. (10)] that $g^{\prime \prime}(0) \neq 0$ implies $g^{\prime}(1)=-\lambda_{0}^{-1}$.

The fact that $g$ should be sought among even functions is justified in the previously quoted works. It can also be supported by the following arguments. 
Denote $h(x)=g(x)-g(x)$ and suppose that, for some $x_{1}>0, h\left(x_{1}\right)>0$. Then

$$
\begin{aligned}
\left|h\left(x_{1}\right)\right| & =\lambda_{0}^{-1}\left|g\left(g\left(-\lambda_{0} x_{1}\right)\right)-g\left(g\left(\lambda_{0} x_{1}\right)\right)\right| \\
& \leqq \lambda_{0}^{-1}\left|h\left(\lambda_{0} x_{1}\right)\right|\left|\int_{0}^{1} d \theta g^{\prime}\left(\theta g\left(-\lambda_{0} x_{1}\right)+(1-\theta) g\left(\lambda_{0} x_{1}\right)\right)\right| .
\end{aligned}
$$

Recall that $g^{\prime}(1)=-\lambda_{0}^{-1}$. For any $\varepsilon>0$ there is a $\delta>0$ such that $x_{1}<\delta$ implies that the last integral is, in modulus, $<(1+\varepsilon) \lambda_{0}^{-1}$ and therefore

$$
\begin{aligned}
& \left|h\left(\lambda_{0} x_{1}\right)\right| \geqq \lambda_{0}^{2}(1+\varepsilon)^{-1}\left|h\left(x_{1}\right)\right|, \\
& \left|h\left(\lambda_{0}^{n} x_{1}\right)\right| \geqq \lambda_{0}^{2 n}(1+\varepsilon)^{-n}\left|h\left(x_{1}\right)\right| .
\end{aligned}
$$

This leads to a contradiction if $h$ is $\mathscr{C}^{3}$, since $h\left(\lambda_{0}^{n} x_{1}\right) \sim \frac{1}{6}\left(\lambda_{0}^{n} x_{1}\right)^{3} h^{\prime \prime \prime}(0)$. In this case there is $\delta>0$ such that $h(x)=0$ for all $x \in[-\delta, \delta]$. Then using repeatedly the above inequality, it follows that $h(x)=0$ everywhere.

\section{Method}

For every $\lambda$ in $I$ (a closed interval around $0.4,0<I<\frac{1}{2}$, to be determined later), we try to find a solution $\chi_{\lambda}$ of (7) within the set $\mathscr{E}_{0}$ of even, $\mathscr{C}^{2}$, concave functions $\phi$ with $\phi(0)=1$. In fact this subset will soon be considerably narrowed down so that functions $\phi$ considered will satisfy: $\phi^{\prime}(0)=0, \phi^{\prime \prime}(0)<0, \phi(1)<0, \phi^{\prime \prime}(x) \leqq 0, \phi^{\prime}(x) \leqq 0$ for all $x \in[0,1]$. Equation (7) implies

$$
\begin{aligned}
& \chi_{\lambda}^{\prime}(x)=-\chi_{\lambda}^{\prime}\left(\chi_{\lambda}(\lambda x)\right) \chi_{\lambda}^{\prime}(\lambda x), \\
& \chi_{\lambda}^{\prime \prime}(x)=-\lambda \chi_{\lambda}^{\prime \prime}\left(\chi_{\lambda}(\lambda x)\right) \chi_{\lambda}^{\prime}(\lambda x)^{2}-\lambda \chi_{\lambda}^{\prime}\left(\chi_{\lambda}(\lambda x)\right) \chi_{\lambda}^{\prime \prime}(\lambda x) .
\end{aligned}
$$

Since we require $\chi_{\lambda}^{\prime \prime}(0)<0, \chi_{\lambda}$ cannot be identically equal to 1 and, moreover, setting $x=0$ in (9) gives

$$
\chi_{\lambda}^{\prime}(1)=-\lambda^{-1}
$$

The method we use consists in substituting the map $\phi \rightarrow \omega(\phi, \lambda)$ with a new mapping $\phi_{1} \rightarrow \tau_{\lambda} \phi_{1}=\phi_{2}$, obtained by solving, for given $\phi_{1}$, the functional equation

$$
\phi_{2}(x)=-\frac{1}{\lambda} \phi_{1}\left(\phi_{2}(\lambda x)\right)+\frac{\phi_{1}(1)}{\lambda}+1 \text {. }
$$

Here $\phi_{1}$ is $\mathscr{C}^{2}$, concave, even, and $\phi_{1}(0)=1$. Then any solution $\phi_{2}$ of $(11)$ also satisfies $\phi_{2}(0)=1$. However (11) implies

$$
\phi_{2}^{\prime \prime}(0)\left[1+\lambda \phi_{1}^{\prime}(1)\right]=0 .
$$

Hence a necessary condition for the Eq. (11) to have a solution with $\phi_{2}^{\prime \prime}(0) \neq 0$ is that

$$
\phi_{1}^{\prime}(1)=-\lambda^{-1}
$$

On the other hand Eq. (11) is not determined since, for any solution $\phi_{2}$ and $0<\varrho \leqq 1, \phi_{2}(\varrho x)$ is also a solution. Thus we define the map $\tau_{\lambda}$ on the set of $\phi_{1}$ 
satisfying $\phi_{1}^{\prime}(1)=-\lambda^{-1}$ by requiring $\phi_{2}$ also to satisfy

$$
\phi_{2}^{\prime}(1)=-\lambda^{-1} \text {. }
$$

It is convenient to define the following new functions

$$
\begin{aligned}
F(x) & =\frac{1}{\lambda}\left[\phi_{1}(1-x)-\phi_{1}(1)\right], \\
G(x) & =\frac{1}{\lambda}\left[\phi_{2}(1-x)-\phi_{2}(1)\right], \quad(0 \leqq x \leqq 1), \\
\psi\left(x^{2}\right) & =1-\phi_{2}(x) .
\end{aligned}
$$

Given $F$, satisfying $F(0)=0, \phi_{1}$ can be reobtained by

$$
\phi_{1}(x)=\lambda[F(1-x)-F(1)]+1
$$

and, similarly

$$
\phi_{2}(x)=\lambda[G(1-x)-G(1)]+1 .
$$

Equation (11) now translates to

$$
\psi(t)=F\left(\psi\left(\lambda^{2} t\right)\right), \quad 0 \leqq t \leqq 1
$$

whence

$$
\psi^{\prime}(t)=\lambda^{2} F^{\prime}\left(\psi\left(\lambda^{2} t\right)\right) \psi^{\prime}\left(\lambda^{2} t\right) .
$$

Because of (16), $\psi$ must satisfy

$$
\psi(0)=0
$$

and hence

$$
\psi^{\prime}(0)=\lambda^{2} F^{\prime}(0) \psi^{\prime}(0)
$$

Noting that $\phi_{2}^{\prime \prime}(0)=-2 \psi^{\prime}(0)$, we recover the necessary condition (12) in the form

$$
F^{\prime}(0)=\lambda^{-2}
$$

and the determining condition (13) takes the form

$$
\psi^{\prime}(1)=\frac{1}{2 \lambda} \text {. }
$$

The map $\tau_{\lambda}$ has now been transformed into a map $T_{\lambda}, T_{\lambda} F=G$, defined as follows :

1) given $F$, a real $\mathscr{C}^{2}$ function on $[0,1]$ satisfying $F(0)=0, F^{\prime}(0)=\frac{1}{\lambda^{2}}$, find a solution $\psi$ of the equations:

$$
\begin{gathered}
\left\{\begin{aligned}
\psi(t) & =F\left(\psi\left(\lambda^{2} t\right)\right) \\
\psi(0) & =0, \psi^{\prime}(0) \neq 0, \psi^{\prime}(1)=(2 \lambda)^{-1},
\end{aligned}\right. \\
G(x)=\frac{1}{\lambda}\left[\psi(1)-\psi\left((1-x)^{2}\right)\right] .
\end{gathered}
$$


A fixed point of $T_{\lambda}$, i.e. an $F$ such that $G=F$ defines a fixed point of $\tau_{\lambda}$ and, consequently a fixed point $\chi_{\lambda}$ of $\omega(\cdot, \lambda)$, and conversely.

In the following sections, we shall obtain subsets of function space where $T_{\lambda}$ is well defined and which are mapped into themselves by $T_{\lambda}$. It will then be proved that, restricted to certain such subsets, equipped with a suitable metric, $T_{\lambda}$ defines a contraction.

\section{More Precise Statement of the Problem and First Estimates}

In this section, $\lambda$ is fixed, with $0<\lambda<1 . F$ will be a $\mathscr{C}^{2}$, concave, increasing function on $[0,1]$, satisfying:

$$
\begin{gathered}
F(0)=0, \quad F^{\prime}(0)=\frac{1}{\lambda^{2}}, \\
0 \leqq F^{\prime}(x), \quad 0 \geqq F^{\prime \prime}(x),
\end{gathered}
$$

hence

$$
0 \leqq F^{\prime}(x) \leqq \frac{1}{\lambda^{2}}, \quad 0 \leqq F(x) \leqq \frac{x}{\lambda^{2}} .
$$

The function $\psi$ must satisfy

$$
\psi(t)=F\left(\psi\left(\lambda^{2} t\right)\right), \quad \psi(0)=0, \quad \psi^{\prime}(0) \neq 0, \quad \psi^{\prime}(1)=(2 \lambda)^{-1} .
$$

We try to determine $\psi$ in the form

$$
\psi(t)=\Psi(\alpha t),
$$

where $\alpha>0$ and $\Psi$ satisfies

$$
\left\{\begin{array}{l}
\Psi(t)=F\left(\Psi\left(\lambda^{2} t\right)\right), \\
\Psi(0)=0, \quad \Psi^{\prime}(0)=1 .
\end{array}\right.
$$

Furthermore there must exist a number $\alpha>0$, in the interval where $\Psi$ exists, such that $\Psi^{\prime}(\alpha)=(2 \alpha \lambda)^{-1}$. In this section we give sufficient conditions for the existence and uniqueness of (30):

Lemma 1. Let $F$ be a real $\mathscr{C}^{2}$ function on $[0, a],(a>0)$, satisfying there

$$
F^{\prime}(x) \geqq 0, \quad F^{\prime \prime}(x) \leqq 0, \quad F(0)=0, \quad F^{\prime}(0)=\lambda^{-2}
$$

and hence

$$
0 \leqq F^{\prime}(x) \leqq \lambda^{-2}, \quad 0 \leqq F(x) \leqq \lambda^{-2} x
$$

Then

(i) There exists a unique $\mathscr{C}^{2}$ function $\Psi$ on $\left[0, a \lambda^{-2}\right]$ such that

$$
\begin{gathered}
0 \leqq \Psi(t) \leqq t, \quad \Psi(0)=0, \quad \Psi^{\prime}(0)=1, \\
\Psi(t)=F\left(\Psi\left(\lambda^{2} t\right)\right) \quad \text { for all } \quad t \in\left[0, a \lambda^{-2}\right],
\end{gathered}
$$


and this function satisfies

$$
0 \leqq \Psi^{\prime}(t) \leqq 1, \quad \Psi^{\prime \prime}(t) \leqq 0 .
$$

(ii) If $F$ is $N$ times continuously differentiable on $[0, a], N>2$, then so is $\Psi$ on $\left[0, a \lambda^{-2}\right]$.

(iii) $\Psi$ depends continuously on $F$ in the $\mathscr{C}^{2}$ topology.

Proof. Let $\mathscr{N}_{1}$ be the convex set consisting of all $\mathscr{C}^{2}$ functions $\Phi$ on $\left[0, \lambda^{-2} a\right]$ verifying $\Phi(0)=0, \Phi^{\prime}(0)=1,0 \leqq \Phi(t) \leqq t$ for all $t$. The mapping

$$
\left(\tau_{F} \Phi\right)(t)=F\left(\Phi\left(\lambda^{2} t\right)\right)
$$

is well defined on $\mathscr{N}_{1}$ and maps $\mathscr{N}_{1}$ into itself. For $\Phi_{1}, \Phi_{2} \in \mathscr{N}_{1}$ denote

Then

$$
d_{0}\left(\Phi_{1}, \Phi_{2}\right)=\sup _{0 \leqq t \leqq a \lambda-2} t^{-2}\left|\Phi_{1}(t)-\Phi_{2}(t)\right| .
$$

$$
d_{0}\left(\tau_{F} \Phi_{1}, \tau_{F} \Phi_{2}\right) \leqq \lambda^{2} d_{0}\left(\Phi_{1}, \Phi_{2}\right) .
$$

This demonstrates that $\tau_{F}$ has at most one fixed point in $\mathscr{N}_{1}$. Let

$$
\mathscr{N}=\left\{\Phi \in \mathscr{C}^{2}\left(\left[0, a \lambda^{-2}\right]\right): 0 \leqq \Phi^{\prime}(t) \leqq 1, \Phi^{\prime \prime}(t) \leqq 0 \text { for all } t, \Phi(0)=0, \Phi^{\prime}(0)=1\right\},
$$

and for $\Phi_{1}, \Phi_{2} \in \mathcal{N}$

$$
d_{1}\left(\Phi_{1}, \Phi_{2}\right)=\sup _{0 \leqq t \leqq a \lambda^{-2}} t^{-1}\left|\Phi_{1}^{\prime}(t)-\Phi_{2}^{\prime}(t)\right| .
$$

Then, using $\left(\tau_{F} \Phi\right)^{\prime}(t)=\lambda^{2} F^{\prime}\left(\Phi\left(\lambda^{2} t\right)\right) \Phi^{\prime}\left(\lambda^{2} t\right)$, one finds

$$
d_{1}\left(\tau_{F} \Phi_{1}, \tau_{F} \Phi_{2}\right) \leqq \lambda^{4} a\left\|F^{\prime \prime}\right\|_{\infty} d_{0}\left(\Phi_{1}, \Phi_{2}\right)+\lambda^{2} d_{1}\left(\Phi_{1}, \Phi_{2}\right)
$$

Denote

$$
\Psi_{0}(t)=t, \Psi_{1}(t)=F\left(\lambda^{2} t\right), \ldots, \Psi_{n}(t)=F\left(\Psi_{n-1}\left(\lambda^{2} t\right)\right)=F \circ F \circ \ldots \circ F\left(\lambda^{2 n} t\right) .
$$

Then, for $n \geqq 1$

$$
\begin{aligned}
& \Psi_{n}^{\prime}(t)=\lambda^{2} F^{\prime}\left(\Psi_{n-1}\left(\lambda^{2} t\right)\right) \Psi_{n-1}^{\prime}\left(\lambda^{2} t\right), \\
& \Psi_{n}^{\prime \prime}(t)=\lambda^{4} F^{\prime \prime}\left(\Psi_{n-1}\left(\lambda^{2} t\right)\right) \Psi_{n-1}^{\prime}\left(\lambda^{2} t\right)^{2}+\lambda^{4} F^{\prime}\left(\Psi_{n-1}\left(\lambda^{2} t\right)\right) \Psi_{n-1}^{\prime \prime}\left(\lambda^{2} t\right) .
\end{aligned}
$$

It follows by induction that $\Psi_{n} \in \mathscr{N}$ for all $n$. Moreover, for $n \geqq 2$,

$$
\begin{aligned}
d_{0}\left(\Psi_{n}, \Psi_{n-1}\right) \leqq & \lambda^{2(n-1)} d_{0}\left(\Psi_{1}, \Psi_{0}\right), \\
d_{1}\left(\Psi_{n}, \Psi_{n-1}\right) \leqq & \lambda^{2 n}(n-1) a\left\|F^{\prime \prime}\right\|_{\infty} d_{0}\left(\Psi_{1}, \Psi_{0}\right) \\
& +\lambda^{2(n-1)} d_{1}\left(\Psi_{1}, \Psi_{0}\right) .
\end{aligned}
$$

Therefore the two sequences $\left\{\Psi_{n}\right\},\left\{\Psi_{n}^{\prime}\right\}$ uniformly converge to $\Psi$ and $\Psi^{\prime}$, respectively, with $\Psi^{\prime}(t)=\frac{d}{d t} \Psi(t)$. Moreover it follows from (33), by induction, that for all $t$,

$$
\left|\Psi_{n}^{\prime \prime}(t)\right| \leqq\left(1-\lambda^{2}\right)^{-1} \lambda^{4}\left\|F^{\prime \prime}\right\|_{\infty}
$$


More generally, suppose that $F \in \mathscr{C}^{N}([0, a]), N \geqq 2$. Then, for $2 \leqq r \leqq N, n \geqq 1$,

$$
\begin{aligned}
\Psi_{n}^{(r)}(t)= & \lambda^{2 r} F^{(r)}\left(\Psi_{n-1}\left(\lambda^{2} t\right)\right) \Psi_{n-1}^{\prime}\left(\lambda^{2} t\right)^{r}+\lambda^{2 r} F^{\prime}\left(\Psi_{n-1}\left(\lambda^{2} t\right)\right) \Psi_{n-1}^{(r)}\left(\lambda^{2} t\right) \\
& +\lambda^{2 r} \mathscr{E}_{r-1}\left(F^{(s)}\left(\Psi_{n-1}\left(\lambda^{2} t\right)\right), \Psi_{n-1}^{(q)}\left(\lambda^{2} t\right)\right),
\end{aligned}
$$

where $\mathscr{E}_{r-1}$ is a polynomial in the quantities indicated, with $1 \leqq q \leqq r-1$, $1 \leqq s \leqq r-1$. It follows from this, by induction on $r$ and $n$, that there is, for each $r=1, \ldots, N$, a number $M_{r}$ (depending only on $F$ ) such that, for all $n$, and all $t$,

$$
\left|\Psi_{n}^{(r)}(t)\right|<M_{r} .
$$

Suppose now that it has been proved, for all $p \leqq r-1,(1 \leqq r \leqq N)$, that $\Psi_{n}^{(p)}$ converges uniformly to a limit (denoted $\Psi^{(p)}$ ) as $n \rightarrow \infty$ (then this limit is the $p^{\text {th }}$ derivative of $\Psi)$. Fix $\varepsilon>0$. By the uniform continuity of $F^{(s)},(0 \leqq s \leqq r)$, and the induction hypothesis, there exists $v(\varepsilon)$ such that, for $m \geqq n \geqq v(\varepsilon)$

$$
\begin{aligned}
& \mid \lambda^{2 r} F^{(r)}\left(\Psi_{n-1}\left(\lambda^{2} t\right)\right) \Psi_{n-1}^{\prime}\left(\lambda^{2} t\right)^{r}+\lambda^{2 r} \mathscr{E}_{r-1}\left(F^{(s)}\left(\Psi_{n-1}\left(\lambda^{2} t\right)\right), \Psi_{n-1}^{(q)}\left(\lambda^{2} t\right)\right) \\
& \quad-\lambda^{2 r} F^{(r)}\left(\Psi_{m-1}\left(\lambda^{2} t\right)\right) \Psi_{m-1}^{\prime}\left(\lambda^{2} t\right)^{r}-\lambda^{2 r} \mathscr{E}_{r-1}\left(F^{(s)}\left(\Psi_{m-1}\left(\lambda^{2} t\right)\right), \Psi_{m-1}^{(q)}\left(\lambda^{2} t\right)\right) \mid<\varepsilon
\end{aligned}
$$

and, as a consequence,

$$
\begin{aligned}
\left\|\Psi_{n}^{(r)}-\Psi_{m}^{(r)}\right\|_{\infty} \leqq & \varepsilon+\lambda^{2 r}\left\|F^{\prime \prime}\right\|_{\infty}\left\|\Psi_{n-1}-\Psi_{m-1}\right\|_{\infty} M_{r} \\
& +\lambda^{2(r-1)}\left\|\Psi_{n-1}^{(r)}-\Psi_{m-1}^{(r)}\right\|_{\infty} .
\end{aligned}
$$

For $m \geqq n \geqq v_{1}(\varepsilon)$, the second term is $\leqq \varepsilon$ and then

$$
\begin{aligned}
\left\|\Psi_{n}^{(r)}-\Psi_{m}^{(r)}\right\|_{\infty} & \leqq 2 \varepsilon+\lambda^{2(r-1)}\left\|\Psi_{n-1}^{(r)}-\Psi_{m-1}^{(r)}\right\| \leqq \ldots \\
& \leqq \frac{2 \varepsilon}{1-\lambda^{2(r-1)}}+2 \lambda^{2(r-1)\left(n-v_{1}(\varepsilon)\right)} M_{r}
\end{aligned}
$$

By choosing $m \geqq n \geqq v_{2}(\varepsilon)$ this can be made smaller than $3 \varepsilon\left(1-\lambda^{2(r-1)}\right)^{-1}$. This proves parts (i) and (ii) of Lemma 1 . We do not give explicitly the proof of (iii) which uses the same methods.

Corollary 2. With the same hypotheses as in Lemma 1, assume that, for some $\kappa>0$, and all $x \in[0, a]$,

$$
0 \leqq-\frac{F^{\prime \prime}(x)}{F^{\prime}(x)} \leqq \kappa
$$

Then, for $t \in\left[0, a \lambda^{-2}\right]$,

$$
\frac{\Psi^{\prime \prime}(t)}{\Psi^{\prime}(t)}=\sum_{n-1}^{\infty} \lambda^{2 n} \Psi^{\prime}\left(\lambda^{2 n} t\right) \frac{F^{\prime \prime}\left(\Psi\left(\lambda^{2 n} t\right)\right)}{F^{\prime}\left(\Psi\left(\lambda^{2 n} t\right)\right)},
$$

where the series on the r.h.s. (which has negative terms) is uniformly convergent.

Proof. The hypotheses imply that $F^{\prime}(x) \geqq \lambda^{-2} e^{-\kappa a}$ for all $x \in[0, a]$ and $\Psi^{\prime}(t) \geqq e^{-n \kappa a} \Psi^{\prime}\left(\lambda^{2 n} t\right)$ for all $t \in\left[0, a \lambda^{-2}\right]$, all $n \geqq 1$ hence, by choosing $n$ large enough, 
$\Psi^{\prime}(t)>\kappa^{\prime}>0$ for all $t \in\left[0, a \lambda^{-2}\right]$. In the expression (valid for all $N \geqq 1$ )

$$
\begin{aligned}
\frac{\Psi^{\prime \prime}(t)}{\Psi^{\prime}(t)} & =\lambda^{2} \Psi^{\prime}\left(\lambda^{2} t\right) \frac{F^{\prime \prime}\left(\Psi\left(\lambda^{2} t\right)\right)}{F^{\prime}\left(\Psi\left(\lambda^{2} t\right)\right)}+\lambda^{2} \frac{\Psi^{\prime \prime}\left(\lambda^{2} t\right)}{\Psi^{\prime}\left(\lambda^{2} t\right)} \\
& =\sum_{n=1}^{N} \lambda^{2 n} \Psi^{\prime}\left(\lambda^{2 n} t\right) \frac{F^{\prime \prime}\left(\Psi\left(\lambda^{2 n} t\right)\right)}{F^{\prime}\left(\Psi\left(\lambda^{2 n} t\right)\right)}+\lambda^{2 N} \frac{\Psi^{\prime \prime}\left(\lambda^{2 N} t\right)}{\Psi^{\prime}\left(\lambda^{2 N} t\right)}
\end{aligned}
$$

the last term tends uniformly to 0 as $N \rightarrow \infty$.

\section{Definition of the Mapping $T_{\lambda}$ and Invariant Subsets}

Given $F$ satisfying the condition of Lemma 1, suppose that there exists $\alpha$ such that $0<\alpha<\lambda^{-2}$ and $\alpha \Psi^{\prime}(\alpha)=(2 \lambda)^{-1}$. Then $T_{\lambda} F=G$ is given by

$$
G(x)=\frac{1}{\lambda}\left[\Psi(\alpha)-\Psi\left(\alpha(1-x)^{2}\right)\right], \quad 0 \leqq x \leqq 1
$$

and satisfies

$$
\begin{gathered}
G^{\prime}(x)=\frac{2 \alpha}{\lambda}(1-x) \Psi^{\prime}\left(\alpha(1-x)^{2}\right) \\
G^{\prime \prime}(x)=-\frac{2 \alpha}{\lambda} \Psi^{\prime}\left(\alpha(1-x)^{2}\right)-\frac{4 \alpha^{2}}{\lambda}(1-x)^{2} \Psi^{\prime \prime}\left(\alpha(1-x)^{2}\right) .
\end{gathered}
$$

If, furthermore $0 \leqq-\frac{F^{\prime \prime}(x)}{F^{\prime}(x)}<\kappa$ for $0 \leqq x \leqq \alpha$, we have (using Corollary 2 )

$$
\frac{G^{\prime \prime}(x)}{G^{\prime}(x)}=\frac{-1}{1-x}-2 \alpha(1-x) \frac{\Psi^{\prime \prime}\left(\alpha(1-x)^{2}\right)}{\Psi^{\prime}\left(\alpha(1-x)^{2}\right)} .
$$

We shall now determine subsets of $\mathscr{C}^{2}([0,1])$ where the above conditions are satisfied and which are mapped into themselves by $T_{\lambda}$. Denote

$$
\begin{gathered}
\mathscr{M}_{\lambda}\left(\ell_{1}, \ell_{3}, c_{1}, c_{3}, A\right)=\left\{F \in \mathscr{C}^{2}([0,1]): F(0)=0,\right. \\
F^{\prime}(0)=\lambda^{-2}, 0 \leqq F^{\prime}(x) \leqq \lambda^{-2}, F^{\prime \prime}(x) \leqq 0, \\
\frac{1}{1-x}-(1-x) \leqq-\frac{F^{\prime \prime}(x)}{F^{\prime}(x)} \leqq \frac{1}{1-x}, \text { for all } x \in[0,1], \text { and } \\
\frac{1}{1-x}-\ell_{1}(1-x)-\ell_{3}(1-x)^{3} \leqq-\frac{F^{\prime \prime}(x)}{F^{\prime}(x)} \leqq \frac{1}{1-x}-c_{1}(1-x)-c_{3}(1-x)^{3} \\
\text { for } 0 \leqq x \leqq A\},
\end{gathered}
$$

where $0<A<1,0 \leqq \ell_{j}, 0 \leqq c_{j},(j=1,3), \ell_{1}+\ell_{3} \leqq 1, c_{1}+c_{3} \leqq 1$.

Let $F$ belong to this set. From $\lambda^{2} F^{\prime}(0)=1$ and

$$
0 \leqq-\frac{F^{\prime \prime}(x)}{F^{\prime}(x)} \leqq \frac{1}{1-x}-c_{1}(1-x)-c_{3}(1-x)^{3}, \quad 0 \leqq x \leqq A,
$$


it follows that, for $0 \leqq x \leqq A$

$$
\begin{aligned}
\lambda^{2} F^{\prime}(x) & \geqq(1-x) \exp \left[\left(c_{1}+c_{3}\right) x-\frac{1}{2}\left(c_{1}+3 c_{3}\right) x^{2}+c_{3}\left(x^{3}-\frac{x^{4}}{4}\right)\right] \equiv H(x) \\
& =(1-x) \exp \left[\frac{c_{1}}{2}\left[1-(1-x)^{2}\right]+\frac{c_{3}}{4}\left[1-(1-x)^{4}\right]\right] .
\end{aligned}
$$

Since the r.h.s. of (41) is $\geqq 0$, the function $H(x)$ is decreasing. On the other hand we have

$$
\Psi^{\prime}(t)=\prod_{n=1}^{\infty}\left(\lambda^{2} F^{\prime}\left(\Psi\left(\lambda^{2 n} t\right)\right)\right)
$$

(Proof.

$$
\Psi^{\prime}(t)=\lambda^{2} F^{\prime}\left(\Psi\left(\lambda^{2} t\right)\right) \Psi^{\prime}\left(\lambda^{2} t\right)=\left[\prod_{n=1}^{N}\left(\lambda^{2} F^{\prime}\left(\Psi\left(\lambda^{2 n} t\right)\right)\right)\right] \Psi^{\prime}\left(\lambda^{2 N} t\right) .
$$

Since $\Psi^{\prime}\left(\lambda^{2 N} t\right) \rightarrow 1$ as $N \rightarrow \infty$, the result follows: note that $\left|\lambda^{2} F^{\prime}\left(\Psi\left(\lambda^{2 n} t\right)\right)-1\right|$ $\leqq \lambda^{2(n-1)}\left\|F^{\prime \prime}\right\|_{\infty}$ so the infinite product is convergent.) Hence, since $H$ is decreasing,

$$
\Psi^{\prime}(t) \geqq \prod_{n=1}^{\infty} H\left(\Psi\left(\lambda^{2 n} t\right)\right) \geqq \prod_{n=1}^{\infty} H\left(\lambda^{2 n} t\right) \quad \text { for } \quad t \in\left[0, A \lambda^{-2}\right] .
$$

Note that, for all $t$, the same holds with $c_{1}=c_{3}=0$, i.e.

$$
\begin{aligned}
\Psi^{\prime}(t) & \geqq \prod_{n=1}^{\infty}\left(1-\lambda^{2 n} t\right) \geqq\left[\prod_{n=1}^{N-1}\left(1-\lambda^{2 n} t\right)\right]\left(1-\lambda^{2 N} t\right)^{\frac{1}{1-\lambda^{2}}} \\
& \geqq\left[\prod_{n=1}^{N-1}\left(1-\lambda^{2 n} t\right)\right]\left(1-\frac{\lambda^{2 N}}{1-\lambda^{2}} t\right) .
\end{aligned}
$$

In particular,

and therefore

$$
\Psi^{\prime}(t) \geqq 1-\frac{\lambda^{2} t}{1-\lambda^{2}},
$$

$$
\Psi(t) \geqq t\left(1-\frac{\lambda^{2} t}{2\left(1-\lambda^{2}\right)}\right) .
$$

Returning to (44), we find, for $0 \leqq t \leqq A \lambda^{-2}$,

$$
\begin{aligned}
\Psi^{\prime}(t) \geqq & {\left[\prod_{n=1}^{\infty}\left(1-\lambda^{2 n} t\right)\right] \exp \frac{\lambda^{2} t}{1-\lambda^{2}}\left[c_{1}+c_{3}-\left(c_{1}+3 c_{3}\right) \frac{\lambda^{2} t}{2\left(1+\lambda^{2}\right)}\right.} \\
& \left.+c_{3} \lambda^{4} t^{2}\left(\frac{1}{1+\lambda^{2}+\lambda^{4}}-\frac{\lambda^{2} t}{4\left(1+\lambda^{2}\right)\left(1+\lambda^{4}\right)}\right)\right] \equiv \Phi(t, \lambda) .
\end{aligned}
$$

Because $H$ is decreasing, $\Phi(t, \lambda)$ is a decreasing function of $t$ for fixed $\lambda$.

From (48) it follows that

$$
\frac{A}{\lambda^{2}} \Psi^{\prime}\left(\frac{A}{\lambda^{2}}\right)>\frac{A}{\lambda^{2}} \Phi\left(\frac{A}{\lambda^{2}}, \lambda\right) .
$$


If the r.h.s. is $>\frac{1}{2 \lambda}$, then, since $t \Psi^{\prime}(t)=0$ at $t=0$ and is continuous, there is at least one value of $t$ in $] 0, A \lambda^{-2}\left[\right.$ for which $t \Psi^{\prime}(t)=\frac{1}{2 \lambda}$ : we denote $\alpha$ the smallest such value. (In fact under the conditions we shall use, it will be shown later that this value is unique.) A sufficient condition for $T_{\lambda}$ to be defined on $\mathscr{M}_{\lambda}\left(\ell_{1}, \ell_{3}, c_{1}, c_{3}, A\right)$ is that:

\section{Criterion 1}

$$
\frac{2 A}{\lambda} \Phi\left(\frac{A}{\lambda^{2}}, \lambda\right)>1
$$

We shall concentrate on a small interval $I$ of variation for $\lambda$, given by

$$
I=\left\{\lambda: 0.152 \leqq \lambda^{2} \leqq 0.166\right\} .
$$

Making $c_{1}=c_{3}=0$, Criterion 1 becomes

$$
\frac{2 A}{\lambda}(1-A)\left(1-\lambda^{2} A\right) \ldots\left(1-\lambda^{2 n} A\right) \ldots \geqq 1 .
$$

For this it is sufficient that, e.g.

$$
\frac{2 A}{\lambda}(1-A)\left(1-\lambda^{2} A\right)\left(1-\frac{\lambda^{4} A}{1-\lambda^{2}}\right) \geqq 1 .
$$

The 1.h.s. of (51) [as well as of (52)] is clearly a decreasing function of $\lambda$ for fixed $A$. The inequality (52) is satisfied for $A=0.32$ and $\lambda^{2}=0.166$ hence for all $\lambda \in I$ : thus we need only consider values of $A \leqq 0.32$, hence $\alpha \lambda^{2} \leqq 0.32$. Under these conditions it follows from (39) and Lemma 1 that

$$
-\frac{G^{\prime \prime}(x)}{G^{\prime}(x)} \leqq \frac{1}{1-x} \quad \text { for } \quad 0 \leqq x<1 .
$$

For future purposes we need smaller values for $A$ (this will later be essential to show that $D T_{\lambda}$ is a contraction) and then it is rather delicate to obtain values for $\ell_{1}, \ell_{3}, c_{1}, c_{3}$, and (sufficiently small) $A$ such that $\mathscr{M}_{\lambda}\left(\ell_{1}, \ell_{3}, c_{1}, c_{3}, A\right)$ is mapped into itself by $T_{\lambda}$. For this, in view of (39), we need upper and lower bounds on $-\frac{\Psi^{\prime \prime}(t)}{\Psi^{\prime}(t)}$.

Upper Bound. Let $c_{1}, c_{3}, A$ and $\lambda$ be fixed with $0 \leqq c_{1}, 0 \leqq c_{3}, c_{1}+c_{3} \leqq 1,0 \leqq A \leqq 1$ and assume $F$ satisfies the hypotheses of Lemma 1 and (41). The r.h.s. of (41) being an increasing function of $x$, using (34) and: $\Psi^{\prime}(t) \leqq 1, \Psi(t) \leqq t$, we find, for $0 \leqq t$ $<A \lambda^{-2}$.

$$
-\frac{\Psi^{\prime \prime}(t)}{\Psi^{\prime}(t)} \leqq \sum_{n=1}^{\infty} \lambda^{2 n}\left[\frac{1}{1-\lambda^{2 n} t}-c_{1}\left(1-\lambda^{2 n} t\right)-c_{3}\left(1-\lambda^{2 n} t\right)^{3}\right] .
$$

Note that for $n \geqq 1, \lambda^{2 n} t \leqq A \leqq 1$, and hence $\left(1-\lambda^{2 n} t\right)^{3} \geqq 1-3 \lambda^{2 n} t$ so that

$$
-\frac{\Psi^{\prime \prime}(t)}{\Psi^{\prime}(t)} \leqq \sum_{n=1}^{\infty}\left[\frac{\lambda^{2 n}}{1-\lambda^{2 n} t}-\lambda^{2 n}\left(c_{1}+c_{3}\right)+\lambda^{4 n} t\left(c_{1}+3 c_{3}\right)\right] \text {. }
$$


Since

$$
\begin{gathered}
\frac{1}{1-\lambda^{2 n} t}=1+\frac{\lambda^{2 n} t}{1-\lambda^{2 n} t} \leqq 1+\frac{\lambda^{2 n} t}{1-\lambda^{2(n-1)} A}, \\
-\frac{\Psi^{\prime \prime}(t)}{\Psi^{\prime}(t)} \leqq \beta_{0}+\beta_{1} t, \quad 0 \leqq t \leqq A \lambda^{-2}
\end{gathered}
$$

with

$$
\begin{aligned}
& \beta_{0}=\frac{\lambda^{2}}{1-\lambda^{2}}\left(1-c_{1}-c_{3}\right) \\
& \beta_{1}=\lambda^{4}\left[\frac{c_{1}+3 c_{3}}{1-\lambda^{4}}+\sum_{n=0}^{N-1} \frac{\lambda^{4 n}}{\left(1-\lambda^{2 n} A\right)}+\frac{\lambda^{4 N}}{\left(1-\lambda^{2 N} A\right)\left(1-\lambda^{4}\right)}\right]
\end{aligned}
$$

(In practice we choose $N=3$.)

Equation (39) now shows that, for $0 \leqq x \leqq 1$,

$$
-\frac{G^{\prime \prime}(x)}{G^{\prime}(x)} \geqq 1-\ell_{1}^{\prime}(1-x)-\ell_{3}^{\prime}(1-x)^{3},
$$

where

$$
\begin{aligned}
\ell_{1}^{\prime} & =\ell_{1}^{\prime}\left(c_{1}, c_{3}, A, \lambda^{2}\right)=\frac{2 A}{\lambda^{2}} \beta_{0}=\frac{2 A}{1-\lambda^{2}}\left(1-c_{1}-c_{3}\right) \\
\ell_{3}^{\prime} & =\ell_{3}^{\prime}\left(c_{1}, c_{3}, A, \lambda^{2}\right)=\frac{2 A^{2}}{\lambda^{4}} \beta_{1} \\
& =2 A^{2}\left[\left(c_{1}+3 c_{3}\right) \frac{1}{1-\lambda^{4}}+\sum_{n=0}^{N-1}\left(\frac{\lambda^{4 n}}{1-\lambda^{2 n} A}\right)+\frac{\lambda^{4 N}}{\left(1-\lambda^{2 N} A\right)\left(1-\lambda^{4}\right)}\right] .
\end{aligned}
$$

The functions $\ell_{1}^{\prime}$ and $\ell_{3}^{\prime}$ are both increasing in $\lambda^{2}$ for fixed $A, c_{1}, c_{3}$. (In practice we choose : $N=3$.)

Lower Bound. Assume $F \in \mathscr{M}_{\lambda}\left(\ell_{1}, \ell_{3}, c_{1}, c_{3}, A\right)$. Then, for $0 \leqq x \leqq A$,

$$
-\frac{F^{\prime \prime}(x)}{F^{\prime}(x)} \geqq 1+x+x^{2}+x^{3}-\ell_{1}(1-x)-\ell_{3}(1-x)^{3} \equiv P(x) \text {. }
$$

Using $\Psi^{\prime}(t) \geqq \Phi(t, \lambda)$ and $\Psi(t) \geqq t\left(1-\frac{\lambda^{2} t}{2\left(1-\lambda^{2}\right)}\right)$, we have, for $t<A \lambda^{-2}$, since $P(x)$ is increasing in $x$,

$$
-\frac{\Psi^{\prime \prime}(t)}{\Psi^{\prime}(t)} \geqq \sum_{n=1}^{\infty} \lambda^{2 n} \Phi\left(\lambda^{2 n} t, \lambda\right) P\left(x_{n}\right)
$$

where

$$
x_{n}=u_{n}\left(1-\frac{\lambda^{2} u_{n}}{2\left(1-\lambda^{2}\right)}\right), \quad u_{n}=\lambda^{2 n} t<A
$$


Using $(1-y)^{3} \geqq 1-3 y$ for $0 \leqq y \leqq 1$, we get

$$
\begin{aligned}
P\left(x_{n}\right) \geqq & \left(1-\ell_{1}-\ell_{3}\right)+\left(u_{n}-\frac{\lambda^{2} u_{n}^{2}}{2\left(1-\lambda^{2}\right)}\right)\left(1+\ell_{1}+3 \ell_{3}\right) \\
& +u_{n}^{2}\left(1-\frac{\lambda^{2} u_{n}}{1-\lambda^{2}}+\frac{\lambda^{4} u_{n}^{2}}{4\left(1-\lambda^{2}\right)^{2}}\right)\left(1-3 \ell_{3}\right) \\
& +u_{n}^{3}\left(1-\frac{3 \lambda^{2} A}{2\left(1-\lambda^{2}\right)}\right)\left(1+\ell_{3}\right) \\
= & \left(1-\ell_{1}-\ell_{3}\right)+u_{n}\left(1+\ell_{1}+3 \ell_{3}\right)+u_{n}^{2}\left(-\frac{\lambda^{2}}{2\left(1-\lambda^{2}\right)}\left(1+\ell_{1}+3 \ell_{3}\right)+1-3 \ell_{3}\right) \\
& +u_{n}^{3}\left[1-\frac{\lambda^{2}}{1-\lambda^{2}}\left(\frac{3 A}{2}+1-\frac{\lambda^{2} u_{n}}{4\left(1-\lambda^{2}\right)}\right)+\ell_{3}\left[1+\frac{3 \lambda^{2}}{1-\lambda^{2}}\left(1-\frac{A}{2}-\frac{\lambda^{2} u_{n}}{4\left(1-\lambda^{2}\right)}\right)\right]\right] .
\end{aligned}
$$

The coefficient of the term in $u_{n}^{3}$ is positive and this term may be dropped. Thus

$$
\begin{aligned}
P\left(x_{n}\right) \geqq & \left(1-\ell_{1}-\ell_{3}\right)+u_{n}\left(1-\frac{\theta \lambda^{2} u_{n}}{2\left(1-\lambda^{2}\right)}\right)\left(1+\ell_{1}+3 \ell_{3}\right) \\
& +u_{n}^{2}\left[1-3 \ell_{3}-\frac{(1-\theta) \lambda^{2}}{2\left(1-\lambda^{2}\right)}\left(1+\ell_{1}+3 \ell_{3}\right)\right] .
\end{aligned}
$$

We choose

$$
Z=\frac{\theta \lambda^{2}}{2\left(1-\lambda^{2}\right)}=\max \left\{0, \frac{\lambda^{2}}{2\left(1-\lambda^{2}\right)}-\frac{1-3 \ell_{3}}{1+\ell_{1}+3 \ell_{3}}\right\}
$$

so that the last term in (63) is positive and can be dropped:

$$
P\left(x_{n}\right) \geqq\left(1-\ell_{1}-\ell_{3}\right)+u_{n}(1-Z A)\left(1+\ell_{1}+3 \ell_{3}\right) .
$$

Note that, according to (64)

We now get, for $t \leqq A \lambda^{-2}$,

$$
Z A \leqq A\left[\frac{\lambda^{2}}{2\left(1-\lambda^{2}\right)}+1\right]<1 .
$$

$$
\left.-\frac{\Psi^{\prime \prime}(t)}{\Psi^{\prime}(t)} \geqq \sum_{n=1}^{\infty} \lambda^{2 n} \Phi\left(\lambda^{2 n} t, \lambda\right)\left[1-\ell_{1}-\ell_{3}\right)+\left(1+\ell_{1}+3 \ell_{3}\right)(1-Z \mathrm{~A}) \lambda^{2 n} \mathrm{t}\right]
$$

and, taking into account the fact that $\Phi(t, \lambda)$ is decreasing in $t$,

$$
\begin{aligned}
-\frac{\Psi^{\prime \prime}(t)}{\Psi^{\prime}(t)} \geqq & \lambda^{2} \Phi\left(\lambda^{2} t, \lambda\right)\left[\left(1-\ell_{1}-\ell_{3}\right)+\left(1+\ell_{1}+3 \ell_{3}\right)(1-Z \mathrm{~A}) \lambda^{2} \mathrm{t}\right] \\
& +\Phi\left(\lambda^{4} t, \lambda\right)\left[\frac{\lambda^{4}}{1-\lambda^{2}}\left(1-\ell_{1}-\ell_{3}\right)+\frac{\lambda^{8} t}{1-\lambda^{4}}\left(1+\ell_{1}+\left(\ell_{3}\right)(1-Z A)\right]\right. \\
\geqq & U+V t,
\end{aligned}
$$


where

$$
\begin{aligned}
& U=\lambda^{2}\left(1-\ell_{1}-\ell_{3}\right)\left[\Phi(A, \lambda)+\frac{\lambda^{2}}{1-\lambda^{2}} \Phi\left(\lambda^{2} A, \lambda\right)\right], \\
& V=\lambda^{4}(1-Z A)\left(1+\ell_{1}+3 \ell_{3}\right)\left[\Phi(A, \lambda)+\frac{\lambda^{4}}{1-\lambda^{4}} \Phi\left(\lambda^{2} A, \lambda\right)\right] .
\end{aligned}
$$

Integrating (65) yields

$$
\Psi^{\prime}(t) \leqq \exp -\left(U t+\frac{V}{2} t^{2}\right), \quad 0 \leqq t<\frac{A}{\lambda^{2}} .
$$

Hence $\alpha$, for which $\alpha=\left(2 \lambda \Psi^{\prime}(\alpha)\right)^{-1}$, must satisfy :

$$
\alpha \geqq \frac{1}{2 \lambda} \exp \alpha\left(U+\alpha \frac{V}{2}\right)
$$

or, denoting $\zeta=2 \lambda \alpha, u=(2 \lambda)^{-1} U, v=\left(8 \lambda^{2}\right)^{-1} V$,

$$
\zeta \geqq \exp \left(u \zeta+v \zeta^{2}\right)
$$

Since, in the cases we consider, $\ell_{1} \geqq c_{1}, \ell_{3} \geqq c_{3}$, so that $\mathscr{M}_{\lambda}\left(\ell_{1}, \ell_{3}, c_{1}, c_{3}, A\right)$ is nonempty, and Criterion 1 is verified so that $\alpha$ exists with $\alpha<A \lambda^{-2}$, the graphs of the functions $\xi \rightarrow \xi$ and $\xi \rightarrow \exp \left(u \xi+v \xi^{2}\right)$ must intersect at two fixed points of the second function. The lower one (denoted $\zeta_{-}$) is stable and is a lower bound for $\zeta$, verifying $\zeta_{-}>1$. For any $\xi_{0}<\zeta_{-}$the sequence $\left\{\xi_{n}\right\}$ given by

$$
\xi_{n}=\exp \left(u \xi_{n-1}+v \xi_{n-1}^{2}\right)
$$

is increasing and tends to $\zeta_{-}$, so that $\zeta_{N}$ is a lower bound for $\zeta_{-}$. In practice, for any $\xi_{0}$ such that $0<\xi_{0}<\zeta_{+}$, if

$$
\xi_{1}=\exp \left(u \xi_{0}+v \xi_{0}^{2}\right)>\xi_{0}
$$

then $\xi_{0}<\xi_{1}<\zeta_{-}$, and $\xi_{1}$ is a lower bound for $\zeta_{-}$. Alternatively, one may choose $\xi_{0}=1$, and stop the sequence (70) at some arbitrary $N$. In this way one obtains a lower bound denoted $\zeta_{\min }$ for $\zeta_{-}$and hence for $\zeta$, so that

$$
\begin{aligned}
& \alpha_{\min } \equiv \frac{1}{2 \lambda} \zeta_{\min }<\alpha, \\
& \alpha_{\min }=\alpha_{\min }\left(\ell_{1}, \ell_{3}, c_{1}, c_{3}, A, \lambda\right) .
\end{aligned}
$$

Hence, for $0 \leqq x<1$,

$$
-\frac{G^{\prime \prime}(x)}{G^{\prime}(x)} \leqq \frac{1}{1-x}-c_{1}^{\prime}(1-x)-c_{3}^{\prime}(1-x)^{3}
$$

where

$$
\begin{aligned}
& c_{1}^{\prime}=c_{1}^{\prime}\left(\ell_{1}, \ell_{3}, c_{1}, c_{3}, A, \lambda\right)=2 \alpha_{\min } U=2 \zeta_{\min } u, \\
& c_{3}^{\prime}=c_{3}^{\prime}\left(\ell_{1}, \ell_{3}, c_{1}, c_{3}, A, \lambda\right)=2 \alpha_{\min }^{2} V=4 \zeta_{\min }^{2} v .
\end{aligned}
$$


In practice, to obtain, for some fixed value of $\lambda$, a set $\mathscr{M}_{\lambda}\left(\ell_{1}, \ell_{3}, c_{1}, c_{3}\right)$ which is mapped into itself by $T_{\lambda}$, one proceeds as follows:

Procedure 0.1) Select $c_{1}, c_{3}$ and $A$ such that (for the value of $\lambda$ under consideration) Criterion 1 is satisfied.

2) Compute $\ell_{1}^{\prime}$ and $\ell_{3}^{\prime}$ and fix $\ell_{1} \geqq \ell_{1}^{\prime}, \ell_{3} \geqq \ell_{3}^{\prime}$, with $0<\ell_{1}+\ell_{3}<1$ (if this is impossible, the trial fails).

3) Compute $U, V, \alpha_{\min }, c_{1}^{\prime}, c_{3}^{\prime}$. The trial is successful if

$$
c_{1}^{\prime} \geqq c_{1}, \quad c_{3}^{\prime} \geqq c_{3} .
$$

Interval Calculations (Procedure 1). It will be necessary to be able to obtain sets $\mathscr{M}_{\lambda}\left(\ell_{1}, \ell_{3}, c_{1}, c_{3}, A\right)$ which are mapped into themselves by $\mathscr{T}_{\lambda}$ for all $\lambda$ in a given interval $J$, say $p \leqq \lambda^{2} \leqq b$. This can be done using the above calculations as follows:

1) Fix $c_{1}, c_{3}$, and $A$ such that Criterion 1 is verified for all $\lambda \in J$ : for this it is sufficient that Criterion 1 be satisfied for $\lambda^{2}=b$.

2) Then for all $F$ satisfying the conditions of Lemma 1 at some $\lambda \in J$ and (41) the upper bound (58) holds. Since $\ell_{1}^{\prime}$ and $\ell_{3}^{\prime}$ are increasing functions of $\lambda^{2}$ we fix

$$
\begin{aligned}
& \ell_{1} \geqq \ell_{1}^{\prime}\left(c_{1}, c_{3}, A, b\right) \\
& \ell_{3} \geqq \ell_{3}^{\prime}\left(c_{1}, c_{3}, A, b\right) .
\end{aligned}
$$

3) Let $F \in \mathscr{M}_{\lambda}\left(\ell_{1}, \ell_{3}, c_{1}, c_{3}, A\right), F^{\prime}(0)=\lambda^{-2}$ with $p \leqq \lambda^{2} \leqq b$. Then the bounds (65-69) hold. We now note that:

a) for fixed $A, c_{1}, c_{3}, \lambda \Phi(A, \lambda)$ and $\lambda \Phi\left(\lambda^{2} A, \lambda\right)$ are increasing functions of $\lambda$ : to see this note first that the exponential appearing in $\Phi(A, \lambda)$ [formula (48)] has been obtained as

$$
\exp \sum_{n=1}^{\infty}\left[\frac{c_{1}}{2}\left[1-\left(1-\lambda^{2 n} A\right)^{2}\right]+\frac{c_{3}}{4}\left[1-\left(1-\lambda^{2 n} A\right)^{4}\right]\right]
$$

and is therefore an increasing function of $\lambda^{2}$. Consider now

$$
\sqrt{\lambda^{2}} \prod_{n=1}^{\infty}\left(1-\lambda^{2 n} A\right) \text {. }
$$

Its logarithmic derivative in $\lambda^{2}$ is :

$$
\begin{aligned}
\frac{1}{2 \lambda^{2}}-\sum_{n=1}^{\infty} \frac{n \lambda^{2 n-2} A}{\left(1-\lambda^{2 n} A\right)} & \geqq \frac{1}{2 \lambda^{2}}-\sum_{n=1}^{\infty} \frac{n \lambda^{2(n-1)} A}{1-\lambda^{2} A} \\
& =\frac{1}{2 \lambda^{2}}-\frac{A}{\left(1-\lambda^{2} A\right)\left(1-\lambda^{2}\right)^{2}} \geqq \frac{1}{2 \lambda^{2}}-\frac{1}{\left(1-\lambda^{2}\right)^{3}}>0 \text { for } \lambda^{2} \leqq 0.166 .
\end{aligned}
$$

The same argument works for $\lambda \Phi\left(\lambda^{2} A, \lambda\right)$.

b) $\lambda(1-Z A)$ is an increasing function of $\lambda$ : The derivative of $Z$ with respect to $\lambda^{2}$ is (for fixed $\ell_{1}, \ell_{3}$ ) equal to 0 if $Z=0$, or to $\frac{A}{2\left(1-\lambda^{2}\right)}\left(1+\frac{\lambda^{2}}{1-\lambda^{2}}\right)$, hence always inferior or equal to this last quantity. 
Hence

$$
\frac{d}{d \lambda^{2}} \lambda(1-Z A) \geqq \frac{1}{2 \lambda}\left(1-\frac{\lambda^{2} A}{2\left(1-\lambda^{2}\right)}-A\right)-\frac{A \lambda}{2\left(1-\lambda^{2}\right)}\left(1+\frac{\lambda^{2}}{1-\lambda^{2}}\right) .
$$

This has the sign of $(1-A)\left(1-\lambda^{2}\right)-\frac{3}{2} \lambda^{2} A-\frac{\lambda^{4} A}{1-\lambda^{2}} \geqq(1-A)\left(1-\lambda^{2}\right)-\frac{5}{2} A \lambda^{2}>0 \quad$ for $\quad \lambda^{2}<0.25, A<0.3$.

c) From this it follows that

$$
\begin{aligned}
& u=\frac{\lambda}{2}\left(1-\ell_{1}-\ell_{3}\right)\left[\Phi(A, \lambda)+\frac{\lambda^{2}}{1-\lambda^{2}} \Phi\left(\lambda^{2} A, \lambda\right)\right] . \\
& v=\frac{\lambda}{8}(1-Z A)\left(1+\ell_{1}+3 \ell_{3}\right) \lambda\left[\Phi(A, \lambda)+\frac{\lambda^{4}}{1-\lambda^{4}} \Phi\left(\lambda^{2} A, \lambda\right)\right],
\end{aligned}
$$

are (for fixed $c_{1}, c_{3}, \ell_{1}, \ell_{3}, A$ ) increasing functions of $\lambda$, hence so is the lowest solution $\zeta_{-}$of $\zeta_{-}=\exp \left(u \zeta+v \zeta^{2}\right)$ [the graph of $\xi \rightarrow \exp \left(u \xi+v \xi^{2}\right)$ rises as $u$ and $v$ increase]. The result of this is that if $\lambda \in J, u, v$ and $\zeta_{\min }$ can be computed with $\lambda^{2}$ replaced by $p$, and therefore also $c_{1}^{\prime}$ and $c_{3}^{\prime}$. However note that $\alpha_{\min }$ must be estimated as

$$
\alpha_{\min }=\frac{\zeta_{\min }}{2 \sqrt{b}} .
$$

Improvement of Constants at Fixed Points (Procedure 3). Let $\lambda, \ell_{1}, \ell_{3}, c_{1}, c_{3}, A$ be fixed such that Criterion 1 is satisfied and $T_{\lambda}$ maps $\mathscr{M}_{\lambda}\left(\ell_{1}, \ell_{3}, c_{1}, c_{3}, A\right)$ into itself. Assume $F_{f} \in \mathscr{M}_{\lambda}\left(\ell_{1}, \ell_{3}, c_{1}, c_{3}, A\right), \alpha_{f}$ is the corresponding value of $\alpha$, and $T_{\lambda} F_{f}=F_{f}$. Then the bounds on $F_{f}$ can be made more precise as follows. Pick $\lambda^{2} \alpha_{\min }<A_{0}<A$. If $\alpha_{f}>A_{o} \lambda^{-2}$, then $\alpha_{\min }$ in the expressions for $c_{1}^{\prime}$ and $c_{3}^{\prime}$ can be replaced by $\lambda^{-2} A_{0}$, leading respectively to $c_{1}^{(1)}$ and $c_{3}^{(1)}$ and the bound

$$
-\frac{F_{f}^{\prime \prime}(x)}{F_{f}^{\prime}(x)} \leqq \frac{1}{1-x}-c_{1}^{(1)}(1-x)-c_{3}^{(1)}(1-x)^{3}
$$

since $F_{f}=T_{\lambda} F_{f}$. Then a new $A_{1}<A$ can be found such that $c_{1}^{(1)}, c_{3}^{(1)}$ and $A_{1}$ satisfy Criterion 1 , so that $\lambda^{2} \alpha_{f} \leqq A_{1}$. This, in turn can be used for a new evaluation of $\ell_{1}^{(2)}$, $\ell_{3}^{(2)}$, and (again using $A_{0} \lambda^{-2}$ instead of $\alpha_{\text {min }}$ ), $c_{1}^{(2)}, c_{3}^{(2)}$ etc. ... One thus constructs a sequence $\ell_{1}^{(n)}, \ell_{3}^{(n)}, c_{1}^{(n)}, c_{3}^{(n)}, A_{n}$. If for a certain $n$ one reaches an $A_{n}<A_{0}$, there is a contradiction so that we must have $\alpha_{f} \leqq A_{0} \lambda^{-2}$. If $A_{n}>A_{0}$ then either $\alpha_{f}>A_{0} \lambda^{-2}$ and then $\alpha_{f} \leqq A_{n} \lambda^{-2}$ and the bounds

$$
\frac{1}{1-x}-\ell_{1}^{(n)}(1-x)-\ell_{3}^{(n)}(1-x)^{3} \leqq-\frac{F_{f}^{\prime \prime}(x)}{F_{f}^{\prime}(x)} \leqq \frac{1}{1-x}-c_{1}^{(n)}(1-x)-c_{3}^{(n)}(1-x)^{3}
$$

hold, or $\alpha_{f} \leqq A_{0} \lambda^{-2}$ so in any case: $\alpha_{f} \leqq A_{n} \lambda^{-2}$. This procedure can be used to obtain a better upper bound for $\alpha_{f}$. Note also that this better bound can be used to improve the bounds on $F_{f}$ : from (55), (58) and the fact that $\ell_{1}^{\prime} \leqq \ell_{1}, \ell_{3}^{\prime} \leqq \ell_{3}$ it follows that

$$
-\frac{F_{f}^{\prime \prime}(x)}{F_{f}^{\prime}(x)} \geqq \frac{1}{1-x}-\ell_{1, f}(1-x)-\ell_{3, f}(1-x)^{3},
$$


where

$$
\ell_{1, f}=\frac{A_{n}}{A} \ell_{1}, \quad \ell_{3, f}=\frac{A_{n}^{2}}{A^{2}} \ell_{3}, \quad A_{f}=A_{n} .
$$

This remark will be used later.

In the sequel we shall say that $\mathscr{M}_{\lambda}\left(\ell_{1}, \ell_{3}, c_{1}, c_{3}, A\right)$ is stable if $c_{1}^{\prime} \geqq c_{1}, c_{2}^{\prime} \geqq c_{3}$, $\ell_{1}^{\prime} \leqq \ell_{1}, \ell_{3}^{\prime} \leqq \ell_{3}$, these new numbers being calculated according to the procedures described above (the Criterion 1 being always assumed to be satisfied). Clearly under these conditions, $\mathscr{M}_{\lambda}\left(\ell_{1}, \ell_{3}, c_{1}, c_{3}, A\right)$ is mapped into itself by $T_{\lambda}$.

\section{Further inequalities}

In this section $F$ is supposed to belong to some stable $\mathscr{M}_{\lambda}\left(\ell_{1}, \ell_{3}, c_{1}, c_{3}, A\right)$ with $A \leqq 0.3$, and to be three times continuously differentiable: then so is $G$ and we have the formulae

$$
\begin{aligned}
\Psi^{\prime \prime \prime}(t)= & \lambda^{6} F^{\prime \prime \prime}\left(\Psi\left(\lambda^{2} t\right)\right) \Psi^{\prime}\left(\lambda^{2} t\right)^{3}+3 \lambda^{6} F^{\prime \prime}\left(\Psi\left(\lambda^{2} t\right)\right) \Psi^{\prime \prime}\left(\lambda^{2} t\right) \Psi^{\prime}\left(\lambda^{2} t\right) \\
& +\lambda^{6} F^{\prime}\left(\Psi\left(\lambda^{2} t\right)\right) \Psi^{\prime \prime \prime}\left(\lambda^{2} t\right), \\
G^{\prime \prime \prime}(x)= & \frac{12 \alpha^{2}}{\lambda}(1-x) \Psi^{\prime \prime}\left(\alpha(1-x)^{2}\right)+\frac{8 \alpha^{3}}{\lambda}(1-x)^{3} \Psi^{\prime \prime \prime}\left(\alpha(1-x)^{2}\right) .
\end{aligned}
$$

Hence

$$
\begin{aligned}
& \frac{\Psi^{\prime \prime \prime}(t)}{\Psi^{\prime}(t)}= \lambda^{4} \frac{F^{\prime \prime \prime}\left(\Psi\left(\lambda^{2} t\right)\right)}{F^{\prime}\left(\Psi\left(\lambda^{2} t\right)\right)} \Psi^{\prime}\left(\lambda^{2} t\right)^{2} \\
&+3 \lambda^{4} \frac{F^{\prime \prime}\left(\Psi\left(\lambda^{2} t\right)\right)}{F^{\prime}\left(\Psi\left(\lambda^{2} t\right)\right)} \Psi^{\prime \prime}\left(\lambda^{2} t\right)+\lambda^{4} \frac{\Psi^{\prime \prime \prime}\left(\lambda^{2} t\right)}{\Psi^{\prime}\left(\lambda^{2} t\right)}, \\
& \frac{G^{\prime \prime \prime}(x)}{G^{\prime}(x)}=6 \alpha \frac{\Psi^{\prime \prime}\left(\alpha(1-x)^{2}\right)}{\Psi^{\prime}\left(\alpha(1-x)^{2}\right)}+4 \alpha^{2}(1-x)^{2} \frac{\Psi^{\prime \prime \prime}\left(\alpha(1-x)^{2}\right)}{\Psi^{\prime}\left(\alpha(1-x)^{2}\right)} .
\end{aligned}
$$

From these, it is useful to form the following combinations (this is the Schwarzian derivative):

$$
\frac{\Psi^{\prime \prime \prime}(t)}{\Psi^{\prime}(t)}-\frac{3}{2}\left(\frac{\Psi^{\prime \prime}(t)}{\Psi^{\prime}(t)}\right)^{2} \equiv(S \Psi)(t)=\lambda^{4}\left[(S F)\left(\Psi\left(\lambda^{2} t\right)\right)\right] \Psi^{\prime}\left(\lambda^{2} t\right)^{2}+\lambda^{4}(S \Psi)\left(\lambda^{2} t\right),
$$

where $(S F)(x) \equiv \frac{F^{\prime \prime \prime}(x)}{F^{\prime}(x)}-\frac{3}{2}\left(\frac{F^{\prime \prime}(x)}{F^{\prime}(x)}\right)^{2}$. Defining similarly $S G$, we have:

Similarly

$$
(S G)(x)=-\frac{3}{2(1-x)^{2}}+4 \alpha^{2}(1-x)^{2}(S \Psi)\left(\alpha(1-x)^{2}\right)
$$

$$
\begin{aligned}
\frac{\Psi^{\prime \prime \prime}(t)}{\Psi^{\prime}(t)}-\left(\frac{\Psi^{\prime \prime}(t)}{\Psi^{\prime}(t)}\right)^{2}= & \lambda^{4} \Psi^{\prime}\left(\lambda^{2} t\right)^{2}\left[\frac{F^{\prime \prime \prime}\left(\Psi\left(\lambda^{2} t\right)\right)}{F^{\prime}\left(\Psi\left(\lambda^{2} t\right)\right)}-\left(\frac{F^{\prime \prime}\left(\Psi\left(\lambda^{2} t\right)\right)}{F^{\prime}\left(\Psi\left(\lambda^{2} t\right)\right)}\right)^{2}\right] \\
& +\lambda^{4} \frac{F^{\prime \prime}\left(\Psi\left(\lambda^{2} t\right)\right)}{F^{\prime}\left(\Psi\left(\lambda^{2} t\right)\right)} \Psi^{\prime \prime}\left(\lambda^{2} t\right) \\
& +\lambda^{4}\left[\frac{\Psi^{\prime \prime \prime}\left(\lambda^{2} t\right)}{\Psi^{\prime}\left(\lambda^{2} t\right)}-\left(\frac{\Psi^{\prime \prime}\left(\lambda^{2} t\right)}{\Psi^{\prime}\left(\lambda^{2} t\right)}\right)^{2}\right]
\end{aligned}
$$


and

$$
\begin{aligned}
\frac{G^{\prime \prime \prime}(x)}{G^{\prime}(x)}-\left(\frac{G^{\prime \prime}(x)}{G^{\prime}(x)}\right)^{2}= & \left\{-\frac{1}{(1-x)^{2}}+2 \alpha \frac{\Psi^{\prime \prime}(t)}{\Psi^{\prime}(t)}\right. \\
& \left.+4 \alpha^{2}(1-x)^{2}\left[\frac{\Psi^{\prime \prime \prime}(t)}{\Psi^{\prime}(t)}-\left(\frac{\Psi^{\prime \prime}(t)}{\Psi^{\prime}(t)}\right)^{2}\right]\right\}_{t=\alpha(1-x)^{2}}
\end{aligned}
$$

We note that formulae (80), (82), (84), (86) continue to hold if $\Psi$ is replaced by $\Psi_{n}$ in the 1.h.s. and by $\Psi_{n-1}$ in the r.h.s. $(n \geqq 1)$. Therefore if we assume that $S F \leqq 0$ on $[0,1]$, it follows by induction on $n$ that $S \Psi_{n} \leqq 0$ and, letting $n \rightarrow \infty, S \Psi \leqq 0$ so, by (85), $S G \leqq 0$. Thus: the subset of $\mathscr{M}_{\lambda}\left(\ell_{1}, \ell_{3}, c_{1}, c_{3}, A\right)$ formed by functions $F$ with $S F \leqq 0,(S F=$ Schwarzian derivative of $F)$, is mapped into itself by $T_{\lambda}$. If $F$ belongs to this subset, then

and

$$
\frac{\Psi^{\prime \prime \prime}(t)}{\Psi^{\prime}(t)} \leqq \frac{3}{2}\left(\frac{\Psi^{\prime \prime}(t)}{\Psi^{\prime}(t)}\right)^{2}
$$

$$
-\frac{G^{\prime \prime \prime}(x)}{G^{\prime}(x)} \geqq-6 \alpha \frac{\Psi^{\prime \prime}(t)}{\Psi^{\prime}(t)}\left[1+t \frac{\Psi^{\prime \prime}(t)}{\Psi^{\prime}(t)}\right],
$$

for $t=\alpha(1-x)^{2}$. But, for $t \leqq \alpha \leqq A \lambda^{-2}$,

$$
0 \leqq-t \frac{\Psi^{\prime \prime}(t)}{\Psi^{\prime}(t)} \leqq \frac{\lambda^{2} t}{1-\lambda^{2} t}+\frac{\lambda^{4} t}{\left(1-\lambda^{4} t\right)\left(1-\lambda^{2}\right)} \leqq \frac{A}{1-A}+\frac{\lambda^{2} A}{\left(1-\lambda^{2} A\right)\left(1-\lambda^{2}\right)}
$$

[see (54)]. For $A \leqq 0.3, \lambda^{2} \leqq 0.166$, this is $<\frac{1}{2}$ so that $G^{\prime \prime \prime}(x) \leqq 0$ and:

$$
-\frac{G^{\prime \prime \prime}(x)}{G^{\prime}(x)} \geqq-3 \alpha \frac{\Psi^{\prime \prime}(t)}{\Psi^{\prime}(t)}, \quad t=\alpha(1-x)^{2} .
$$

Hence (for such values of the constants) the subset of functions in $\mathscr{M}$, with negative Schwarzian derivative is mapped into the smaller subset of those with negative third derivative. Assume now that $F^{\prime \prime \prime} \leqq 0$. Then we have

$$
\sigma(t) \equiv \frac{\Psi^{\prime \prime \prime}(t)}{\Psi^{\prime}(t)}-\left(\frac{\Psi^{\prime \prime}(t)}{\Psi^{\prime}(t)}\right)^{2} \leqq 0
$$

Indeed, from (86) and $F^{\prime \prime \prime} \leqq 0$, it follows that

$$
\begin{array}{r}
\sigma(t) \leqq \lambda^{4} \sigma\left(\lambda^{2} t\right)+\lambda^{4} \Psi^{\prime}\left(\lambda^{2} t\right) \frac{F^{\prime \prime}\left(\Psi\left(\lambda^{2} t\right)\right)}{F^{\prime}\left(\Psi\left(\lambda^{2} t\right)\right)} \\
\cdot\left[-\frac{F^{\prime \prime}\left(\Psi\left(\lambda^{2} t\right)\right)}{F^{\prime}\left(\Psi\left(\lambda^{2} t\right)\right)} \Psi^{\prime}\left(\lambda^{2} t\right)+\frac{\Psi^{\prime \prime}\left(\lambda^{2} t\right)}{\Psi^{\prime}\left(\lambda^{2} t\right)}\right] .
\end{array}
$$

We claim the last term in (93) is $\leqq 0$ :

$$
\begin{aligned}
& -\frac{F^{\prime \prime}\left(\Psi\left(\lambda^{2} t\right)\right)}{F^{\prime}\left(\Psi\left(\lambda^{2} t\right)\right)} \Psi^{\prime}\left(\lambda^{2} t\right)+\frac{\Psi^{\prime \prime}\left(\lambda^{2} t\right)}{\Psi^{\prime}\left(\lambda^{2} t\right)} \\
& =-\frac{F^{\prime \prime}\left(\Psi\left(\lambda^{2} t\right)\right)}{F^{\prime}\left(\Psi\left(\lambda^{2} t\right)\right)} \Psi^{\prime}\left(\lambda^{2} t\right)-\sum_{n=1}^{\infty} \lambda^{2 n} \Psi^{\prime}\left(\lambda^{2 n+2} t\right)\left[-\frac{F^{\prime \prime}\left(\Psi\left(\lambda^{2 n+2} t\right)\right)}{F^{\prime}\left(\Psi\left(\lambda^{2 n+2} t\right)\right)}\right]
\end{aligned}
$$


since $-\frac{F^{\prime \prime}(x)}{F^{\prime}(x)}$ is an increasing function, this is bounded below by

and

$$
-\frac{F^{\prime \prime}\left(\Psi\left(\lambda^{2} t\right)\right)}{F^{\prime}\left(\Psi\left(\lambda^{2} t\right)\right)}\left[\Psi^{\prime}\left(\lambda^{2} t\right)-\frac{\lambda^{2}}{1-\lambda^{2}}\right]
$$

$$
\Psi^{\prime}\left(\lambda^{2} t\right)-\frac{\lambda^{2}}{1-\lambda^{2}} \geqq 1-\frac{\lambda^{2}}{1-\lambda^{2}}\left(\lambda^{2} t+1\right) \geqq 1-\frac{2 \lambda^{2}}{1-\lambda^{2}}>0 .
$$

Thus, for all $t \in\left[0, \lambda^{-2}\right]$

hence

$$
\sigma(t) \leqq \lambda^{4} \sigma\left(\lambda^{2} t\right)
$$

$$
\sup _{t} \sigma(t) \leqq \lambda^{4} \sup _{t} \sigma(t), \quad\left(1-\lambda^{4}\right) \sup _{t} \sigma(t) \leqq 0 . \quad \text { Q.E.D. }
$$

We also need, in the sequel, bounds on $\sigma(t)$, on $\frac{F^{\prime \prime \prime}}{F^{\prime}}-\left(\frac{F^{\prime \prime}}{F^{\prime}}\right)^{2}$ and on $\Psi^{\prime \prime \prime}(t)$ : these are obtained as follows. Suppose that $F^{\prime \prime \prime} \leqq 0, F \in \mathscr{M}_{\lambda}\left(\ell_{1}, \ell_{3}, c_{1}, c_{3}, A\right)$, stable, and that, furthermore,

$$
\sup _{0 \leqq x \leqq A}\left[-\frac{F^{\prime \prime \prime}(x)}{F^{\prime}(x)}+\left(\frac{F^{\prime \prime}(x)}{F^{\prime}(x)}\right)^{2}\right] \leqq L .
$$

Then, by (86), for all $t \in\left[0, \lambda^{-2} A\right]$

$$
-\sigma(t) \leqq \lambda^{4} L-\lambda^{4} \sigma\left(\lambda^{2} t\right)
$$

so

$$
\left(1-\lambda^{4}\right) \sup _{0 \leqq t \leqq A \lambda-2}(-\sigma(t)) \leqq \lambda^{4} L
$$

i.e.

$$
\sup _{0 \leqq t \leqq A \lambda-2}|\sigma(t)| \leqq \frac{\lambda^{4}}{1-\lambda^{4}} L
$$

Hence, by (87)

$$
\begin{aligned}
-\frac{G^{\prime \prime \prime}(x)}{G^{\prime}(x)}+\left(\frac{G^{\prime \prime}(x)}{G^{\prime}(x)}\right)^{2} \leqq & -2 \alpha \frac{\Psi^{\prime \prime}\left(\alpha(1-x)^{2}\right)}{\Psi^{\prime}\left(\alpha(1-x)^{2}\right)} \\
& +\frac{1}{(1-x)^{2}}+\frac{4 \alpha^{2} \lambda^{4} L}{1-\lambda^{4}}(1-x)^{2}
\end{aligned}
$$

and, for $0 \leqq x \leqq A$, the r.h.s. of (96) is bounded by

$$
2 \alpha \beta_{0}+2 \alpha^{2} \beta_{1}(1-x)^{2}+\frac{1}{(1-x)^{2}}+\frac{4 \alpha^{2} \lambda^{4} L}{1-\lambda^{4}}(1-x)^{2},
$$

where we have used (55). Since $\alpha \leqq A \lambda^{-2}$, this is bounded by

$$
\ell_{1}+\left(\ell_{3}+\frac{4 A^{2} L}{1-\lambda^{4}}\right) y+\frac{1}{y}
$$


where $(1-A)^{2} \leqq y=(1-x)^{2} \leqq 1$. This is a convex function of $y$ whose maximum is therefore reached at one of the ends of the interval $\left[(1-A)^{2}, 1\right]$. Let $y_{1}$ be the value where the maximum is reached. Then the r.h.s. of (96) is bounded by (97) with $y=y_{1}$ and, therefore, a sufficient condition for $G$ to verify the same bound (94) as $F$ is

$$
L \geqq \ell_{1}+\left(\ell_{3}+\frac{4 A^{2} L}{1-\lambda^{4}}\right) y_{1}+\frac{1}{y_{1}}
$$

or

$$
L \geqq\left[1-\frac{4 A^{2}}{1-\lambda^{4}} y_{1}\right]^{-1}\left[\ell_{1}+\ell_{3} y_{1}+y_{1}^{-1}\right]
$$

and, in particular, [denoting $(1-A)^{2}=y_{0}$ ],

$$
L=\max _{y=1 \text { or } y_{0}}\left[1-\frac{4 A^{2}}{1-\lambda^{4}} y\right]^{-1}\left[\ell_{1}+\ell_{3} y+y^{-1}\right] .
$$

In all the cases which we need in the sequel the maximum is obtained for $y=y_{0}$. This means that the subset of the functions $F \in \mathscr{M}_{\lambda}\left(\ell_{1}, \ell_{3}, c_{1}, c_{3}, A\right)$ obeying $F^{\prime \prime \prime} \leqq 0$ and (94), with $L$ given by (98) or (99), is mapped into itself by $T_{\lambda}$; note also that (95) holds for such $F$.

In the same spirit, denote $\Psi_{ \pm}^{\prime \prime \prime}(t)=\sup \left\{0, \pm \Psi^{\prime \prime \prime}(t)\right\}$. Then, for $t \leqq A \lambda^{-2}$

$$
\begin{aligned}
\frac{\Psi_{-}^{\prime \prime \prime}(t)}{\Psi^{\prime}(t)} & \leqq \lambda^{4} K_{F}+\lambda^{4} \frac{\Psi_{-}^{\prime \prime \prime}\left(\lambda^{2} t\right)}{\Psi^{\prime}\left(\lambda^{2} t\right)} \\
K_{F} & =\sup _{0 \leqq x \leqq 1}-\frac{F^{\prime \prime \prime}(x)}{F^{\prime}(x)}
\end{aligned}
$$

and, denoting

$$
M=\sup _{0 \leqq t \leqq A \lambda^{-2}} \frac{\Psi_{-}^{\prime \prime \prime}(t)}{\Psi^{\prime}(t)}, K_{G}=\sup _{0 \leqq x \leqq 1}-\frac{G^{\prime \prime \prime}(x)}{G^{\prime}(x)}
$$

we find:

$$
\begin{aligned}
M & \leqq \frac{\lambda^{4}}{1-\lambda^{4}} K_{F}, \\
K_{G} & \leqq 3\left(2 \alpha \beta_{0}+2 \alpha^{2} \beta_{1}\right)+\frac{4 \alpha^{2} \lambda^{4}}{1-\lambda^{4}} K_{F} .
\end{aligned}
$$

Hence

$$
K_{G} \leqq 3\left(\ell_{1}+\ell_{3}\right)+\frac{4 A^{2}}{1-\lambda^{4}} K_{F} .
$$

By the same calculation, (94) implies:

$$
K_{G} \leqq 3\left(\ell_{1}+\ell_{3}\right)+4 A^{2} L\left(1-\lambda^{4}\right)^{-1} .
$$


The inequality $K_{G} \leqq K_{F}$ will be satisfied if

$$
K_{F} \geqq\left(1-\frac{4 A^{2}}{1-\lambda^{4}}\right)^{-1} 3\left(\ell_{1}+\ell_{3}\right) .
$$

As a consequence the subset in $\mathscr{M}_{\lambda}\left(\ell_{1}, \ell_{3}, c_{1}, c_{3}, A\right)$ such that

$$
\left|F^{\prime \prime \prime}(x) / F^{\prime}(x)\right| \leqq 3\left(\ell_{1}+\ell_{3}\right)\left(1-4 A^{2} /\left(1-\lambda^{4}\right)\right)^{-1}
$$

is preserved by $T_{\lambda}$. Moreover, at a fixed point, since $K_{G}=K_{F}$ and $\alpha \leqq \lambda^{-2} A_{f}$, (see Sect. 5), with $\ell_{1, f}$ and $\ell_{3, f}$ as in (79), we find

$$
\begin{aligned}
K_{F(\text { fixed point })} & \leqq 3\left(\ell_{1, f}+\ell_{3, f}\right)\left(1-4 A_{f}^{2} /\left(1-\lambda^{4}\right)\right)^{-1} \\
M_{\text {fixed point }} & \leqq 3 \lambda^{4}\left(\ell_{1, f}+\ell_{3, f}\right)\left(1-\lambda^{4}-4 A_{f}^{2}\right)^{-1} .
\end{aligned}
$$

Finally, we have

$$
\begin{aligned}
\left(1-\lambda^{4}\right) \sup _{0 \leqq t \leqq A \lambda^{-2}} \frac{\Psi_{+}^{\prime \prime \prime}(t)}{\Psi^{\prime}(t)} & \leqq 3 \lambda^{4}\left[\sup _{0 \leqq x \leqq A}-\frac{F^{\prime \prime}(x)}{F^{\prime}(x)}\right]\left[\sup _{0 \leqq t \leqq A \lambda^{-2}}\left|\Psi^{\prime \prime}(t)\right|\right] \\
& \leqq 3 \lambda^{4}\left[\frac{1}{1-A}-c_{1}(1-A)-c_{3}(1-A)^{3}\right]\left(\beta_{0}+A \lambda^{-2} \beta_{1}\right) .
\end{aligned}
$$

where we have used (55) and $\left|\Psi^{\prime}(t)\right| \leqq 1$. Since $\beta_{0}+A \lambda^{-2} \beta_{1}=\frac{\lambda^{2}}{2 A}\left(\ell_{1}^{\prime}+\ell_{3}^{\prime}\right) \leqq \frac{\lambda^{2}}{2 A}$ $\left(\ell_{1}+\ell_{3}\right),(104)$ also yields

$$
\sup _{0 \leqq t \leqq A \lambda^{-2}} \frac{\Psi_{+}^{\prime \prime \prime}(t)}{\Psi^{\prime}(t)}<\frac{3 \lambda^{6}}{2 A\left(1-\lambda^{4}\right)}\left[\frac{1}{(1-A)}-c_{1}(1-A)-c_{3}(1-A)^{3}\right]\left(\ell_{1}+\ell_{3}\right) .
$$

The same bound holds for $\Psi_{+}^{\prime \prime \prime}(t)$, since $\Psi^{\prime}(t) \leqq 1$.

We now adopt the following notation: $\mathscr{S}_{\lambda}\left(\ell_{1}, \ell_{3}, c_{1}, c_{3}, A\right)$ denotes the set of $\mathscr{C}^{3}$ functions on $[0,1]$ verifying the conditions :

1) $0 \leqq F(x) \leqq \lambda^{-2}, 0 \leqq F^{\prime}(x) \leqq \lambda^{-2}$ for all $x \in[0,1], F(0)=0, F^{\prime}(0)=\lambda^{-2}$.

2) For all $x \in[0,1]$

$$
\begin{aligned}
(1-x)^{-1}-\ell_{1}(1-x)-\ell_{3}(1-x)^{3} \leqq & -\frac{F^{\prime \prime}(x)}{F^{\prime}(x)} \leqq(1-x)^{-1} \\
& -c_{1}(1-x)-c_{3}(1-x)^{3} .
\end{aligned}
$$

The constants $\ell_{1}, \ldots, c_{3}$ are always supposed to satisfy $0 \leqq c_{1} \leqq \ell_{1}, 0 \leqq c_{3} \leqq \ell_{3}$, $\ell_{1}+\ell_{3}<1$.

3) For all $x \in[0,1], F^{\prime \prime \prime}(x) \leqq 0$. Moreover for $0 \leqq x \leqq A$

$$
-\frac{F^{\prime \prime \prime}(x)}{F^{\prime}(x)}+\left(\frac{F^{\prime \prime}(x)}{F^{\prime}(x)}\right)^{2} \leqq L
$$

with $L$ given by (99).

We say, for short, that $\mathscr{S}_{\lambda}\left(\ell_{1}, \ell_{3}, c_{1}, c_{3}, A\right)$ is stable if

1) $c_{1}, c_{3}, A$ satisfy Criterion 1 .

2) $\ell_{1} \geqq \ell_{1}^{\prime}\left(c_{1}, c_{3}, A, \lambda^{2}\right), \ell_{3} \geqq \ell_{3}^{\prime}\left(c_{1}, c_{3}, A, \lambda^{2}\right)$.

3) $c_{1}^{\prime}\left(\ell_{1}, \ell_{3}, c_{1}, c_{3}, A, \lambda\right) \geqq c_{1}, c_{3}^{\prime}\left(\ell_{1}, \ell_{3}, c_{1}, c_{3}, A, \lambda\right) \geqq c_{3}$. 
The content of Sects. 4-7 can be summarized by: if $\mathscr{S}_{\lambda}\left(\ell_{1}, \ell_{3}, c_{1}, c_{3}, A\right)$ is stable, then it is mapped into itself by $T_{\lambda}$.

We note, furthermore, that formulae (99), (102) are, for fixed $\ell_{1}, \ell_{3}$, and $A$, increasing functions of $\lambda$. Let $J=\left\{\lambda: p \leqq \lambda^{2} \leqq b\right\}$. We define $\mathscr{S}_{J}\left(\ell_{1}, \ell_{3}, c_{1}, c_{3}\right)$ by the conditions

1) $0 \leqq F(x), 0 \leqq F^{\prime}(x), F(0)=0, b^{-1} \leqq F^{\prime}(0) \leqq p^{-1}$,

2) same as for $\mathscr{S}_{\lambda}$,

3) same as for $\mathscr{S}_{\lambda}$, but with $\lambda^{2}$ replaced, in formulae (99), (102), by $b$.

$\mathscr{S}_{J}\left(\ell_{1}, \ell_{3}, c_{1}, c_{3}\right)$ is then said to be stable if

1) $c_{1}, c_{3}, A$ satisfy Criterion 1 ,

2) $\ell_{1} \geqq \ell_{1}^{\prime}\left(c_{1}, c_{3}, A, b\right), \ell_{3} \geqq \ell_{3}^{\prime}\left(c_{1}, c_{3}, A, b\right)$,

3) $c_{1}^{\prime}\left(\ell_{1}, \ell_{3}, c_{1}, c_{3}, A, \sqrt{p}\right) \geqq c_{1}$, $c_{3}^{\prime}\left(\ell_{1}, \ell_{3}, c_{1}, c_{3}, A, \sqrt{p}\right) \geqq c_{3}$.

In this case, for each $\lambda \in J$, the set $\mathscr{S}_{J} \cap\left\{F: F^{\prime}(0)=\lambda^{-2}\right\}$ is mapped into itself by $T_{\lambda}$ for every $\lambda \in J$.

\section{Analyticity}

For fixed $\lambda$, let $F$ belong to a stable $\mathscr{S}_{\lambda}\left(\ell_{1}, \ell_{3}, c_{1}, c_{3}, A\right)$. We note that, by formula (38) the corresponding $G$ verifies

$$
0 \leqq-G^{\prime \prime}(x) \leqq \frac{2 \alpha}{\lambda} \leqq \frac{2 A}{\lambda^{3}} \text { for all } x \in[0,1] .
$$

We now make the assumption that, for all $x \in[0,1]$ and all $n \geqq 1$,

$$
\left|\frac{1}{n !}\left(\frac{d}{d x}\right)^{n} F(x)\right| \leqq C B^{n}=\frac{1}{\lambda^{2}} B^{n-1},
$$

where $B$ is some positive constant, and $C B=\lambda^{-2}$.

Then, for all $n, \Psi_{n}$ (defined in the proof of Lemma 1) is $\mathscr{C}^{\infty}$ and we propose to find an estimate of the form $\forall m, \forall r \geqq 1$,

$$
\left|\frac{1}{r !}\left(\frac{d}{d t}\right)^{r} \Psi_{m}(t)\right| \leqq \Lambda M^{r}=M^{r-1}, 0 \leqq t \leqq \lambda^{-2},
$$

where $M>0$ is independent of $m, \Lambda M=1$. We use the method of majorizing power series. Note that (108) holds for $\Psi_{0}(t)=t$ and suppose it holds for all $\Psi_{m}$ with $m \leqq N-1$. Then, given $t_{0} \in\left[0, \lambda^{-2}\right]$

$$
\begin{aligned}
\Psi_{N}\left(t_{0}+s\right)-\Psi_{N}\left(t_{0}\right) & =F\left(\Psi_{N-1}\left(\lambda^{2} t_{0}\right)+z\right)-F\left(\Psi_{N-1}\left(\lambda^{2} t_{0}\right)\right)=\sum_{m=1}^{\infty} a_{m} z^{m}, \\
a_{m} & =\frac{1}{m !} D^{m} F\left(\Psi_{N-1}\left(\lambda^{2} t_{0}\right)\right), \\
z & =\Psi_{N-1}^{\prime}\left(\lambda^{2} t_{0}+\lambda^{2} s\right)-\Psi_{N-1}\left(\lambda^{2} t_{0}\right)=\sum_{r=1}^{\infty} \lambda^{2 r} s^{r} c_{r} \\
c_{r} & =\frac{1}{r !} D^{r} \Psi_{N-1}\left(\lambda^{2} t_{0}\right) .
\end{aligned}
$$


Hence

$$
\begin{aligned}
\Psi_{N}\left(t_{0}+s\right)-\Psi_{N}\left(t_{0}\right) & =\sum_{n=1}^{\infty} v_{n} \lambda^{2 n} S^{n}, \\
v_{n} & =\sum_{p=1}^{n} a_{p} \sum_{\substack{r_{1}, \ldots, r_{p} \geqq 1 \\
r_{1}+\ldots+r_{p}=n}} c_{r_{1}} \ldots c_{r_{p}} .
\end{aligned}
$$

Taking into account the preceding bounds shows that:

where

$$
\left|v_{n}\right| \leqq V_{n}, n \geqq 1
$$

$$
\begin{aligned}
\sum_{n=1}^{\infty} V_{n} s^{n} & =\Phi(U(s)) \\
\Phi(z) & =\sum_{n=1}^{\infty} C B^{n} z^{n}=\frac{C}{1-B z}-C \\
U(s) & =\sum_{n=1}^{\infty} \Lambda M^{n} s^{n}=\frac{\Lambda}{1-M s}-\Lambda=\frac{s}{1-M s}, \\
\Phi(U(s)) & =\frac{C B}{B+M}\left[\frac{1}{1-(B+M) s}-1\right]
\end{aligned}
$$

so that,

$$
V_{n}=C B(B+M)^{n-1} .
$$

Hence $\Psi_{N}$ also satisfies (108) if, for all $n \geqq 1$,

$$
C B \lambda^{2 n}(B+M)^{n-1} \leqq M^{n-1},
$$

i.e. taking into account $\lambda^{2} C B=1$,

$$
\lambda^{2}(B+M) \leqq M
$$

i.e.

$$
M \geqq \frac{\lambda^{2}}{1-\lambda^{2}} B .
$$

Thus, if this condition is satisfied, for all $r \geqq 1$, and all $m,(108)$ holds. By Vitali's theorem, it follows that $\left\{\Psi_{n}\right\}$ converges to $\Psi$ in the sense of analytic functions so that $\Psi$ is $\mathscr{C}^{\infty}$ on $\left[0, \lambda^{-2}\right]$ and also satisfies

$$
\left|\frac{1}{r !} D^{r} \Psi(t)\right| \leqq \Lambda M^{r}=M^{r-1} \text { for all } t \in\left[0, \lambda^{-2}\right] \text { and all } r \geqq 1 .
$$

We now ask under which conditions the function $G$ also satisfies the bounds (107). First note that $|G(x)| \leqq \lambda^{-2},\left|G^{\prime}(x)\right| \leqq \lambda^{-2},\left|G^{\prime \prime}(x)\right| \leqq 2 A \lambda^{-3}, \lambda^{2}\left|G^{\prime \prime \prime}(x)\right| \leqq[$ r.h.s. of (102)]. Hence if we suppose

$$
B \geqq A \lambda^{-1}, B^{2} \geqq \frac{1}{6}[\text { r.h.s. of }(102)],
$$

there remains to determine whether, for all $x \in[0,1]$

$$
\left|\frac{1}{n !} D^{n} G(x)\right| \leqq C B^{n}=\frac{1}{\lambda^{2}} B^{n-1} \quad \text { for } \quad n \geqq 4 \text { ? }
$$


We apply once more the principle of majorizing power series : given $x_{0} \in[0,1]$,

$$
\begin{aligned}
\Psi\left(\alpha\left(1-x_{0}-z\right)^{2}\right)-\Psi\left(\alpha\left(1-x_{0}\right)^{2}\right) & =\sum_{n=1}^{\infty} c_{n}(\alpha w)^{n}, \\
w & =-2\left(1-x_{0}\right) z+z^{2} \\
c_{n} & =\frac{1}{n !} D^{n} \Psi\left(\alpha\left(1-x_{0}\right)^{2}\right) .
\end{aligned}
$$

The power series expressing $w$ in terms of $z$ is majorized by $2 z+z^{2}$. Hence if $\zeta_{n}=\frac{1}{n !} D^{n} G\left(x_{0}\right)$, we have

$$
\begin{aligned}
\left|\zeta_{n}\right| & \leqq Z_{n} \\
\sum_{n=1}^{\infty} Z_{n} z^{n} & =\frac{1}{\lambda} U\left(\alpha\left(2 z+z^{2}\right)\right) \\
& =\frac{\Lambda}{\lambda}\left[\frac{1}{1-M \alpha\left(2 z+z^{2}\right)}-1\right] .
\end{aligned}
$$

We denote

$$
\begin{aligned}
& 1-2 M \alpha z-M \alpha z^{2}=(1+\sigma z)(1-\varrho z), \text { where } \sigma>0 \text { and }: \\
& \varrho-\sigma=2 M \alpha, \varrho \sigma=M \alpha,(\varrho+\sigma)^{2}=4 M^{2} \alpha^{2}+4 M \alpha .
\end{aligned}
$$

Then

$$
\begin{gathered}
\sum_{n=1}^{\infty} Z_{n} z^{n}=\frac{\Lambda}{\lambda(\varrho+\sigma)}\left[\frac{\varrho}{1-\varrho z}+\frac{\sigma}{1+\sigma z}\right]-\frac{\Lambda}{\lambda}, \\
Z_{n}=\frac{\Lambda}{\lambda(\varrho+\sigma)}\left[\varrho^{n+1}-(-\sigma)^{n+1}\right] .
\end{gathered}
$$

Since $0<\sigma<\varrho, \varrho^{n}(\varrho+\sigma)-\varrho^{n+1}+(-\sigma)^{n+1}=\sigma\left(\varrho^{n}-(-\sigma)^{n}\right) \geqq 0$, so $Z_{n} \leqq \Lambda \lambda^{-1} \varrho^{n}$, with

$$
\varrho=M \alpha\left[1+\left(1+\frac{1}{M \alpha}\right)^{1 / 2}\right], M=B \lambda^{2}\left(1-\lambda^{2}\right)^{-1}=\Lambda^{-1}
$$

A sufficient condition for (113) to be satisfied is therefore $\frac{B \lambda}{M}\left(\frac{\varrho}{B}\right)^{n} \leqq 1$ for all $n \geqq 4$.

This will be satisfied if and only if $(\varrho / B) \leqq 1$ and $B \lambda M^{-1}(\varrho / B)^{4} \leqq 1$. With $M=\lambda^{2}\left(1-\lambda^{2}\right)^{-1} B$, the conditions to be satisfied [apart from (112)], are

$$
\begin{gathered}
\frac{\alpha \lambda^{2}}{1-\lambda^{2}}\left[1+\left(1+\frac{1}{M \alpha}\right)^{1 / 2}\right] \equiv \kappa \leqq 1 \\
\kappa \leqq\left(\frac{\lambda}{1-\lambda^{2}}\right)^{1 / 4}
\end{gathered}
$$


For $\lambda<0.5$, the r.h.s. of (116) is inferior to 1 , so (116) implies (115). A necessary condition for (116) to be possible is clearly

$$
\frac{2 \alpha \lambda^{2}}{1-\lambda^{2}} \leqq\left(\frac{\lambda}{1-\lambda^{2}}\right)^{1 / 4}
$$

which holds if

$$
(2 A)^{4}<\lambda\left(1-\lambda^{2}\right)^{3} .
$$

(This is always verified if e.g. $A<0.32,0.152 \leqq \lambda^{2} \leqq 0.25$.) Then (110) is equivalent to

$$
\frac{1}{M \alpha} \leqq\left[\frac{\lambda^{1 / 4}\left(1-\lambda^{2}\right)^{3 / 4}}{\alpha \lambda^{2}}-1\right]^{2}-1
$$

or

$$
B \geqq \frac{\left(1-\lambda^{2}\right)}{\alpha \lambda^{2}}\left[\left[\frac{\lambda^{1 / 4}\left(1-\lambda^{2}\right)^{3 / 4}}{\alpha \lambda^{2}}-1\right]^{2}-1\right]^{-1} .
$$

It is easy to verify that this is, for fixed $\alpha \lambda^{2}$, a decreasing function of $\lambda$ and, for fixed $\lambda$, an increasing function of $\alpha \lambda^{2}$ (in the range of values we consider). Thus a list of sufficient conditions for (113) are

$$
\begin{aligned}
B & \geqq \frac{1-\lambda^{2}}{A}\left[\left[\frac{\lambda^{1 / 4}\left(1-\lambda^{2}\right)^{3 / 4}}{A}-1\right]^{2}-1\right]^{-1}, \\
B & \geqq A \lambda^{-1}, \\
B^{2} & \geqq \frac{1}{2}\left(\ell_{1}+\ell_{3}\right)+\frac{2}{3} A^{2} L\left(1-\lambda^{4}\right)^{-1} .
\end{aligned}
$$

[For these conditions to hold for all $\lambda$ in the interval $p \leqq \lambda^{2} \leqq b, \lambda$ must be replaced by $\sqrt{p}$ in (119) and (120) and by $\sqrt{b}$ in (121).] If $B_{0}$ is the smallest number verifying these consitions, we see that, for all $B \geqq B_{0}$, the subset of functions $F$ in $\mathscr{S}_{\lambda}\left(\ell_{1}, \ell_{3}, c_{1}, c_{3}, A\right)$, which obey (107) is mapped into itself by $T_{\lambda}$. In fact it is easy to verify, by the same calculations, that if $B$ satisfies

$$
\begin{aligned}
B & \geqq \frac{1-\lambda^{2}}{A}\left[\left[\frac{\left(1-\lambda^{2}\right)^{3 / 4}\left(\tau^{3} \lambda\right)^{1 / 4}}{A}-1\right]^{2}-1\right]^{-1} \\
\tau B & \geqq A \lambda^{-1} \\
\tau^{2} B^{2} & \geqq[\text { r.h.s. of }(121)],
\end{aligned}
$$

with $0<\tau<1$ and $\left(1-\lambda^{2}\right)^{3} \tau^{3} \lambda-(2 A)^{4}>0$, then

$$
\left|\frac{1}{n !} D^{n} G(x)\right| \leqq \lambda^{-2}(\tau B)^{n-1} \quad \text { for all } n \geqq 1 \text {. }
$$

The numerical results, to be described in Sect. 10, give $B_{0}<1.8$ and a corresponding $\frac{1}{M_{0} \alpha}>1.79$ for all cases of interest. For a given $B$, let $\mathscr{A}_{B}$ denote the set of $\mathscr{C}^{\infty}$ functions on $(0,1]$, which satisfy the bounds (107). This is a convex set in 
$\mathscr{C}^{2}([0,1])$ and any $F \in \mathscr{A}_{B}$ is analytic in

$$
\left\{z \in \mathbb{C}: d(z,[0,1])<B^{-1}\right\} .
$$

In particular, if $\mathscr{S}_{\lambda}\left(\ell_{1}, \ell_{3}, c_{1}, c_{3}, A\right)$ is stable, and $B \geqq B_{0}$ (as determined above) the set

$$
\mathscr{A}_{B} \cap \mathscr{S}_{\lambda}\left(\ell_{1}, \ell_{3}, c_{1}, c_{3}, A\right) \text {, }
$$

is mapped into itself by $T_{\lambda}$. This set is non-empty by Sect. 8 , and compact in the $\mathscr{C}^{2}$ topology: indeed it is relatively compact by Ascoli's theorem, and closed because, by Vitali's theorem any Cauchy sequence in this set in the $\mathscr{C}^{2}$ topology converges to an analytic function satisfying the bounds (107). Similarly $\mathscr{A}_{B} \cap \mathscr{S}_{J}\left(\ell_{1}, \ell_{3}, c_{1}, c_{3}, A\right)$ is stable.

\section{Non Triviality of $\mathscr{S}_{\lambda} \cap \mathscr{A}_{B}$}

To verify that, for a suitable choice of the constants, $\mathscr{I}_{\lambda}\left(\ell_{1}, \ell_{3}, c_{1}, c_{3}, A\right)$ is non empty, we consider the function $F$ defined by

$$
\begin{gathered}
F(0)=0, \quad F^{\prime}(0)=\lambda^{-2}, \\
-\frac{F^{\prime \prime}(x)}{F^{\prime}(x)}=\frac{1}{1-x}-c_{1}(1-x)-c_{3}(1-x)^{3},
\end{gathered}
$$

and consequently,

$$
\begin{gathered}
\lambda^{2} F^{\prime}(x)=(1-x) \exp \left\{\frac{c_{1}}{2}\left[1-(1-x)^{2}\right]+\frac{c_{3}}{4}\left[1-(1-x)^{4}\right]\right\}, \\
\frac{F^{\prime \prime \prime}(x)}{F^{\prime}(x)}-\left(\frac{F^{\prime \prime}(x)}{F^{\prime}(x)}\right)^{2}=-\frac{1}{(1-x)^{2}}-c_{1}-3 c_{3}(1-x)^{2} .
\end{gathered}
$$

This last expression, (the derivative of $F^{\prime \prime} / F^{\prime}$ ) is negative and for $0 \leqq x \leqq A$, bounded in modulus by

$$
\frac{1}{y_{0}}+c_{1}+3 c_{3} y_{0}, \quad y_{0}=(1-A)^{2} .
$$

For all cases of interest here (see Tables 1 and 2), this expression is inferior to $L$ as given by (99). From (124) and (126) we get:

$$
\frac{F^{\prime \prime \prime}(x)}{F^{\prime}(x)}=-3 c_{1}-5 c_{3}(1-x)^{2}+(1-x)^{2}\left[c_{1}+c_{3}(1-x)^{2}\right]^{2}
$$

The last term is positive but bounded in modulus by 1 and therefore by $c_{1}+c_{3}(1-x)^{2}$ so that the total expression is negative. (In particular $\left.\left|F^{\prime \prime \prime}(x)\right|<\left(3 c_{1}+5 c_{3}\right) \lambda^{-2}\right)$. Hence $-F^{\prime \prime}$ takes its maximum at $x=1$, with the value

$$
-F^{\prime \prime}(1)=\lambda^{-2} \exp \left(\frac{c_{1}}{2}+\frac{c_{3}}{4}\right) .
$$

In order to estimate the successive derivatives of $F$, we denote $z=1-x$ and

$$
\lambda^{2} F^{\prime}(x)=h(z)=z \exp \left[\frac{c_{1}}{2}\left(1-z^{2}\right)+\frac{c_{3}}{4}\left(1-z^{4}\right)\right] .
$$


Table 1

\begin{tabular}{|c|c|c|c|c|c|c|c|c|c|c|c|}
\hline$\lambda^{2}$ & $A$ & $c_{1}$ & $c_{1}^{\prime}>$ & $c_{3}$ & $c_{3}^{\prime}>$ & $\ell_{1}$ & $\ell_{1}^{\prime}<$ & $\ell_{3}$ & $\ell_{3}^{\prime}<$ & Norm $\leqq$ & $\zeta_{\min } \geqq$ \\
\hline 0.152 & 0.243 & 0.22 & 0.22004 & 0.224 & 0.2241 & 0.3187 & 0.318651 & 0.2668 & 0.266741 & 0.814 & 1.1806 \\
\hline 0.154 & 0.2453 & 0.215 & 0.2157 & 0.228 & 0.2288 & 0.3231 & 0.323008 & 0.2734 & 0.273314 & 0.834 & 1.1795 \\
\hline 0.156 & 0.248 & 0.208 & 0.2099 & 0.232 & 0.2331 & 0.3291 & 0.3290996 & 0.2808 & 0.280737 & 0.858 & 1.1773 \\
\hline 0.158 & 0.2505 & 0.204 & 0.205 & 0.237 & 0.2377 & 0.3327 & 0.332612 & 0.2886 & 0.288562 & 0.88 & 1.1759 \\
\hline 0.16 & 0.253 & 0.2 & 0.2003 & 0.242 & 0.2422 & 0.3362 & 0.336129 & 0.2966 & 0.296538 & 0.903 & 1.1743 \\
\hline 0.162 & 0.2555 & 0.194 & 0.1946 & 0.246 & 0.2466 & 0.3415 & 0.34148 & 0.304 & 0.303996 & 0.928 & 1.1723 \\
\hline 0.164 & 0.2585 & 0.187 & 0.18703 & 0.25 & 0.2509 & 0.3482 & 0.348172 & 0.3128 & 0.312776 & 0.958 & 1.1691 \\
\hline 0.165 & 0.2595 & 0.185 & 0.18505 & 0.2532 & 0.25324 & 0.3492 & 0.349191 & 0.3166 & 0.316592 & 0.969 & 1.1686 \\
\hline
\end{tabular}

\section{Table 2}

\begin{tabular}{|c|c|c|c|c|c|c|c|c|c|c|c|}
\hline$J$ & $A$ & $c_{1}$ & $c_{1}^{\prime}>$ & $c_{3}$ & $c_{3}^{\prime}>$ & $\ell_{1}$ & $\ell_{1}^{\prime}<$ & $\ell_{3}$ & $\ell_{3}^{\prime}<$ & Norm $\leqq$ & $\zeta_{\min } \geqq$ \\
\hline $0.152 \leqq \lambda^{2} \leqq 0.161$ & 0.26 & 0.16 & 0.1624 & 0.224 & 0.22454 & 0.3818 & 0.381788 & 0.302 & 0.301934 & 0.981 & 1.1472 \\
\hline $0.161 \leqq \lambda^{2} \leqq 0.164$ & 0.261 & 0.172 & 0.1727 & 0.243 & 0.2438 & 0.3653 & 0.365276 & 0.3145 & 0.314438 & 0.987 & 1.1587 \\
\hline $0.164 \leqq \lambda^{2} \leqq 0.165$ & 0.261 & 0.176 & 0.1769 & 0.248 & 0.25 & 0.3601 & 0.360087 & 0.3172 & 0.317192 & 0.987 & 1.1631 \\
\hline
\end{tabular}


We wish to verify that, for some $B$, for all $n \geqq 1$ and $0<z \leqq 1$.

$$
\left|D^{n} h(z)\right|=\left|D^{n+1} \lambda^{2} F(1-x)\right| \leqq(n+1) ! B^{n} .
$$

The Cauchy inequalities give, for all $z \in[0,1], R>1, n \geqq 0$,

$$
\left|D^{n} h(z)\right| \leqq n !\|h\|_{R}(R-1)^{-n}, \quad\|h\|_{R}=\sup _{\substack{w \in \mathbb{C} \\|w| \leqq R}}|h(w)| .
$$

It suffices to verify that:

$$
\begin{aligned}
B & \geqq \frac{1}{2} \exp \left(\frac{c_{1}}{2}+\frac{c_{3}}{4}\right), \\
B^{2} & \geqq \frac{1}{6}\left(3 c_{1}+5 c_{3}\right),
\end{aligned}
$$

and, for all $n \geqq 3$, some $\mathrm{R}>1$,

$$
(n+1) B^{n} \geqq\|h\|_{R}(R-1)^{-n} .
$$

This last condition is equivalent to

$$
B \geqq \max \left\{\frac{1}{R-1}, \frac{1}{R-1}\left(\frac{\|h\|_{R}}{4}\right)^{1 / 3}\right\} .
$$

Noting that

$$
\|h\|_{R} \leqq R \exp \left[\frac{c_{1}}{2}\left(1+R^{2}\right)+\frac{c_{3}}{4}\left(1+R^{4}\right)\right],
$$

we find that, choosing e.g. $c_{1} \leqq 0.25, c_{3} \leqq 0.3, R=2$, all these conditions are satisfied for $B>1.5$. This covers all the cases we need.

We have now verified that, for all the values of the constants relevant to our purposes, the function $F$ satisfies the conditions for being in $\mathscr{S}_{\lambda}\left(\ell_{1}, \ell_{3}\right.$, $\left.c_{1}, c_{3}, A\right) \cap \mathscr{A}_{B}$.

\section{Map Derivative}

We now introduce a new set of functions

$$
\begin{aligned}
& \mathscr{H}_{\lambda}^{\prime}\left(\ell_{1}, \ell_{3}, c_{1}, c_{3}, A\right) \\
& \quad=\left\{s \in \mathscr{C}^{1}([0, A]): s(x)=\frac{F^{\prime \prime}(x)}{F^{\prime}(x)}, F \in \mathscr{S}_{\lambda}\left(\ell_{1}, \ell_{3}, c_{1}, c_{3}, A\right)\right\} .
\end{aligned}
$$

This subset of $\mathscr{C}^{1}([0, A])$ is in one-to-one correspondence with $\mathscr{S}_{\lambda}\left(\ell_{1}, \ell_{3}, c_{1}, c_{3}, A\right)$ $[0, A]$ through the map $k$ :

$$
\begin{aligned}
(k F)(x) & =\frac{F^{\prime \prime}(x)}{F^{\prime}(x)} \\
\left(k^{-1} s\right)(x) & =\int_{0}^{x} d y \lambda^{-2} \exp \int_{0}^{y} s(z) d z .
\end{aligned}
$$


$\mathscr{H}_{\lambda}^{\prime}$ consists just of those functions $s$ satisfying

$$
\begin{aligned}
& \frac{1}{1-x}-\ell_{1}(1-x)-\ell_{3}(1-x)^{3} \leqq-s(x) \leqq \frac{1}{1-x}-c_{1}(1-x)-c_{3}(1-x)^{3}, \\
& 0 \leqq-\left[s^{\prime}(x)+s(x)^{2}\right] \\
& \quad-s^{\prime}(x) \leqq L .
\end{aligned}
$$

We consider $\mathscr{H}_{\lambda}^{\prime}$ as a subset of the space $\mathscr{C}([0, A])$ of continuous functions on $[0, A]$ equipped with the norm

$$
\|s\|=\sup _{0 \leqq x \leqq A}\left|(1-x)^{-1} s(x)\right| .
$$

We define a map $\bar{T}_{\lambda}: \mathscr{H}_{\lambda}^{\prime} \rightarrow \mathscr{H}_{\lambda}^{\prime}$ as

$$
\bar{T}_{\lambda}=k T_{\lambda} k^{-1}
$$

(in other words, $\bar{T}_{\lambda}$ maps $\frac{F^{\prime \prime}}{F^{\prime}}$ onto $\frac{G^{\prime \prime}}{G^{\prime}}$ with the preceding notations). Since the map $F \rightarrow \Psi$ is continuous in the $\mathscr{C}^{2}$ topology, and since $\alpha$, defined by $2 \alpha \lambda \Psi^{\prime}(\alpha)=1$, continuously depends on $\Psi$, the map $T_{\lambda}$ is continuous in the $\mathscr{C}^{2}$ topology in a neighborhood of $\mathscr{S}_{\lambda}\left(\ell_{1}, \ell_{3}, c_{1}, c_{3}, A\right)$, so that $\bar{T}_{\lambda}$ is continuous in some neighborhood $U$ of $\mathscr{H}_{\lambda}^{\prime}$ in $\mathscr{C}([0, A])$. We propose to estimate the Fréchet derivative of this map at points of $\mathscr{H}_{\lambda}^{\prime}$.

Recall that the Fréchet derivative $D E(s)$ of a map $s \rightarrow E(s)$ is (when it exists) a linear operator $\left[\right.$ on $\mathscr{C}^{1}([0, A])$ in our case $]$ such that, for any $u$

$$
\lim _{\|u\| \rightarrow 0}\|u\|^{-1}[E(s+u)-E(s)-D E(s) u]=0 .
$$

To make the formulae more transparent, we denote the vector $u$ occurring in this formula by $\delta\left(\frac{F^{\prime \prime}}{F^{\prime}}\right)$. Similarly

$$
\begin{aligned}
\delta F^{\prime}(x) & =F^{\prime}(x) \int_{0}^{x} \delta\left(\frac{F^{\prime \prime}(y)}{F^{\prime}(y)}\right) d y=F^{\prime}(x) \int_{0}^{x} u(y) d y, \\
\delta F(x) & =\int_{0}^{x} \delta F^{\prime}(y) d y .
\end{aligned}
$$

We also denote

$$
\|\delta F\|_{2}=\|u\|=\sup _{0 \leqq x \leqq A}(1-x)^{-1}\left|\delta\left(\frac{F^{\prime \prime}(x)}{F^{\prime}(x)}\right)\right|
$$

Note that

$$
\begin{gathered}
\left|\frac{\delta F^{\prime}(x)}{F^{\prime}(x)}\right| \leqq\|\delta F\|_{2} \int_{0}^{x}(1-y) d y=Q(x)\|\delta F\|_{2}, \\
Q(x) \equiv x\left(1-\frac{x}{2}\right) .
\end{gathered}
$$


Defining

$$
\|\delta F\|_{1}=\sup _{0 \leqq x \leqq A} Q(x)^{-1}\left|\frac{\delta F^{\prime}(x)}{F^{\prime}(x)}\right|
$$

we have $\|\delta F\|_{1} \leqq\|\delta F\|_{2}$ and

$$
\begin{gathered}
|\delta F(x)| \leqq \lambda^{-2}\|\delta F\|_{1} \int_{0}^{x} Q(y) d y=\lambda^{-2}\|\delta F\|_{1} P(x), \\
P(x) \equiv \frac{x^{2}}{2}\left(1-\frac{x}{3}\right),
\end{gathered}
$$

Setting $\|\delta F\|_{0}=\sup _{0 \leqq x \leqq A} \lambda^{2} P(x)^{-1}|\delta F(x)|$ yields :

$$
\|\delta F\|_{0} \leqq\|\delta F\|_{1} \leqq\|\delta F\|_{2} .
$$

We shall start by defining the linear operator $A_{0}$ by $\left(\mathrm{A}_{0} \delta F\right)(t) \equiv \delta \Psi(t)$ as the solution of the equation

$$
\delta \Psi(t)=\delta F\left(\Psi\left(\lambda^{2} t\right)\right)+F^{\prime}\left(\Psi\left(\lambda^{2} t\right)\right) \delta \Psi\left(\lambda^{2} t\right) .
$$

Note that the map $v \rightarrow w$ defined by

$$
w(t)=\delta F\left(\Psi\left(\lambda^{2} t\right)\right)+F^{\prime}\left(\Psi\left(\lambda^{2} t\right)\right) v\left(\lambda^{2} t\right)
$$

is a contraction in the distance defined by the norm $\||v|\|_{0}=\sup _{0 \leqq x \leqq A \lambda^{-2}} t^{-2}|v(t)|$ since

$$
t^{-2}\left|w_{1}(t)-w_{2}(t)\right| \leqq \lambda^{-2} t^{-2}\left|v_{1}\left(\lambda^{2} t\right)-v_{2}\left(\lambda^{2} t\right)\right| \leqq\left.\lambda^{2}||\left|v_{1}-v_{2}\right|\right|_{0}
$$

so that the solution of $(142)$ is unique and given by

$$
\delta \Psi(t)=\sum_{n=1}^{\infty}\left[\prod_{1 \leqq j \leqq n-1} F^{\prime}\left(\Psi\left(\lambda^{2 j} t\right)\right)\right] \delta F\left(\Psi\left(\lambda^{2 n} t\right)\right) .
$$

We now look for a better estimate of $\delta \Psi(t)$ : from (142) it follows that

$$
\begin{aligned}
\left|t^{-2} \delta \Psi(t)\right| & \equiv v_{0}(t) \leqq t^{-2} \lambda^{-2} P\left(\Psi\left(\lambda^{2} t\right)\right)\|\delta F\|_{0}+\lambda^{2} v_{0}\left(\lambda^{2} t\right) \\
& \leqq w_{0}(t)+\lambda^{2} v_{0}\left(\lambda^{2} t\right), \\
w_{0}(t) & =\frac{\lambda^{2}}{2}\left(1-\frac{\lambda^{2} t}{3}\right)\|\delta F\|_{0},
\end{aligned}
$$

[where we have used $\Psi\left(\lambda^{2} \mathrm{t}\right) \leqq \lambda^{2} t$ and the fact that $P(x)$ is increasing when $0 \leqq x$ $\leqq 1]$. Iterating this yields [as would also (143)].

$$
v_{0}(t) \leqq \sum_{n=0}^{\infty} \lambda^{2 n} w_{0}\left(\lambda^{2 n} t\right)=\left[\frac{\lambda^{2}}{2\left(1-\lambda^{2}\right)}-\frac{\lambda^{4} t}{6\left(1-\lambda^{4}\right)}\right]\|\delta F\|_{0} .
$$

Thus, for all $t \in\left[0, A \lambda^{-2}\right]$,

$$
\begin{gathered}
|\delta \Psi(t)| \leqq S_{0}(t)\|\delta F\|_{0}, \\
S_{0}(t) \equiv \frac{\lambda^{2} t^{2}}{2\left(1-\lambda^{4}\right)}\left[1+\lambda^{2}-\frac{\lambda^{2} t}{3}\right] .
\end{gathered}
$$


Similarly let $\frac{\delta \Psi^{\prime}(t)}{\Psi^{\prime}(t)}$ denote the solution of

$$
\frac{\delta \Psi^{\prime}(t)}{\Psi^{\prime}(t)}=\frac{\delta F^{\prime}\left(\Psi\left(\lambda^{2} t\right)\right)}{F^{\prime}\left(\Psi\left(\lambda^{2} t\right)\right)}+\frac{F^{\prime \prime}\left(\Psi\left(\lambda^{2} t\right)\right)}{F^{\prime}\left(\Psi\left(\lambda^{2} t\right)\right)} \delta \Psi\left(\lambda^{2} t\right)+\frac{\delta \Psi^{\prime}\left(\lambda^{2} t\right)}{\Psi^{\prime}\left(\lambda^{2} t\right)} .
$$

This equation is obtained by differentiating (142) and using $\Psi^{\prime}(t)$ $=\lambda^{2} F^{\prime}\left(\Psi\left(\lambda^{2} t\right)\right) \Psi^{\prime}\left(\lambda^{2} t\right)$. It is clear that the series obtained by formally interating this equation and suitably substituting (143) coincides with the series obtained by differentiating term by term (143). Hence the uniform convergence of the iteration of (146) will prove [together with the absolute convergence of (143)] that its solution is the derivative of $\delta \Psi(t)$ divided by $\Psi^{\prime}(t)$. The proof of this fact will follow the same line as above: denote $v_{1}(t)=\left[t^{\prime}(t)\right]^{-1}\left|\delta \Psi^{\prime}(t)\right|$ then (146) implies

$$
\begin{aligned}
v_{1}(t) \leqq & t^{-1} Q\left(\lambda^{2} t\right)\|\delta F\|_{1} \\
& +t^{-1} S_{0}\left(\lambda^{2} t\right)\left[\frac{1}{1-\lambda^{2} t}-c_{1}\left(1-\lambda^{2} t\right)-c_{3}\left(1-\lambda^{2} t\right)^{3}\right]\|\delta F\|_{0} \\
& +\lambda^{2} v_{1}\left(\lambda^{2} t\right)
\end{aligned}
$$

where we have used (139), (144), and the bound (40) on $\frac{F^{\prime \prime}}{F^{\prime}}$. Hence

$$
v_{1}(t) \leqq \lambda^{2}\left(1-\frac{\lambda^{2} t}{2}\right)\|\delta F\|_{1}+\frac{\lambda^{6} t}{2\left(1-\lambda^{4}\right)} f(t)\|\delta F\|_{1}+\lambda^{2} v_{1}\left(\lambda^{2} t\right)
$$

with

$$
f(t)=\left(1+\lambda^{2}-\frac{\lambda^{4} t}{3}\right)\left[\left(1-\lambda^{2} t\right)^{-1}-c_{1}\left(1-\lambda^{2} t\right)-c_{3}\left(1-\lambda^{2} t\right)^{3}\right] .
$$

We have

$$
\begin{aligned}
f^{\prime}(t)= & -\frac{\lambda^{4}}{3}\left[\left(1-\lambda^{2} t\right)^{-1}-c_{1}\left(1-\lambda^{2} t\right)-c_{3}\left(1-\lambda^{2} t\right)^{3}\right] \\
& +\left(1+\lambda^{2}-\frac{\lambda^{4} t}{3}\right)\left[\lambda^{2}\left(1-\lambda^{2} t\right)^{-2}+c_{1} \lambda^{2}+3 c_{3} \lambda^{2}\left(1-\lambda^{2} t\right)^{2}\right] \\
\geqq & \lambda^{2}\left(1-\lambda^{2} t\right)^{-2}\left(1+\frac{2}{3} \lambda^{2}\right)>0 .
\end{aligned}
$$

Thus $f$ is increasing so $f\left(\lambda^{2 n} t\right) \leqq f(t)$ and the iteration of (148) gives

$$
\begin{aligned}
v_{1}(t) & \leqq \sum_{n=1}^{\infty}\left\{\lambda^{2 n}-\frac{\lambda^{4 n} t}{2}+\frac{\lambda^{2} \lambda^{4 n} t}{2\left(1-\lambda^{4}\right)} f(t)\right\}\|\delta F\|_{1} \\
& =\left[\frac{\lambda^{2}}{1-\lambda^{2}}-\frac{\lambda^{4} t}{2\left(1-\lambda^{4}\right)}+\frac{\lambda^{6} t}{2\left(1-\lambda^{4}\right)^{2}} f(t)\right]\|\delta F\|_{1} .
\end{aligned}
$$

Hence [majorizing $f(t)$ by $\left.f\left(A \lambda^{-2}\right)\right]$,

$$
\begin{gathered}
\left|\frac{d \Psi^{\prime}(t)}{\Psi^{\prime}(t)}\right| \leqq S_{1}(t)\|\delta F\|_{1}, \\
S_{1}(t)=\frac{\lambda^{2} t}{1-\lambda^{2}}\left[1-\frac{\lambda^{2} t}{2\left(1-\lambda^{4}\right)}\left(1-\lambda^{2}-\lambda^{2} N\left(1-\frac{\lambda^{2} A}{3\left(1+\lambda^{2}\right)}\right)\right)\right]
\end{gathered}
$$


with

$$
N=(1-A)^{-1}-c_{1}(1-A)-c_{3}(1-A)^{3} .
$$

From this and the equation

$$
\delta \alpha=-\alpha\left[1+\alpha \frac{\Psi^{\prime \prime}(\alpha)}{\Psi^{\prime}(\alpha)}\right]^{-1} \frac{\delta \Psi^{\prime}(\alpha)}{\Psi^{\prime}(\alpha)}
$$

it follows that

$$
\begin{aligned}
\|\delta F\|_{1}^{-1}|\delta \alpha| & \leqq \alpha\left[1-\beta_{0} \alpha-\beta_{1} \alpha^{2}\right]^{-1} S_{1}(\alpha) \\
& \leqq \frac{A}{\lambda^{2}}\left(1-\beta_{0} \frac{A}{\lambda^{2}}-\beta_{1} \frac{A^{2}}{\lambda^{4}}\right)^{-1} S_{1}\left(A \lambda^{-2}\right) \leqq \frac{A}{\lambda^{2}} h_{3} \\
h_{3} & =\left(1-\frac{\ell_{1}}{2}-\frac{\ell_{3}}{2}\right)^{-1} S_{1}\left(A \lambda^{-2}\right)
\end{aligned}
$$

We now investigate in the same manner

$$
\begin{aligned}
\delta\left(\frac{\Psi^{\prime \prime}(t)}{\Psi^{\prime}(t)}\right)= & \lambda^{2} \delta \Psi^{\prime}\left(\lambda^{2} t\right) \frac{F^{\prime \prime}\left(\Psi\left(\lambda^{2} t\right)\right)}{F^{\prime}\left(\Psi\left(\lambda^{2} t\right)\right)} \\
& +\left.\lambda^{2} \Psi^{\prime}\left(\lambda^{2} t\right) \delta\left(\frac{F^{\prime \prime}(x)}{F^{\prime}(x)}\right)\right|_{x=\Psi\left(\lambda^{2} t\right)} \\
& +\lambda^{2} \Psi^{\prime}\left(\lambda^{2} t\right)\left[\left(\frac{F^{\prime \prime}(x)}{F^{\prime}(x)}\right)\right]_{x=\Psi\left(\lambda^{2} t\right)} \delta \Psi\left(\lambda^{2} t\right) \\
& +\lambda^{2} \delta\left(\frac{\Psi^{\prime \prime}\left(\lambda^{2} t\right)}{\Psi^{\prime}\left(\lambda^{2} t\right)}\right)
\end{aligned}
$$

Denoting $v_{2}(t)=\left|\delta\left(\frac{\Psi^{\prime \prime}(t)}{\Psi^{\prime}(t)}\right)\right|$ gives, for all $t \in\left[0, A \lambda^{-2}\right]$,

$$
\begin{aligned}
v_{2}(t) \leqq & \lambda^{2} S_{1}\left(\lambda^{2} t\right)\left[\left(1-\lambda^{2} t\right)^{-1}-c_{1}\left(1-\lambda^{2} t\right)-c_{3}\left(1-\lambda^{2} t\right)^{3}\right]\|\delta F\|_{1} \\
& +\lambda^{2} \Psi^{\prime}\left(\lambda^{2} t\right)\left(1-\Psi\left(\lambda^{2} t\right)\right)\|\delta F\|_{2} \\
& +\lambda^{2} L S_{0}\left(\lambda^{2} t\right)\|\delta F\|_{0} \\
& +\lambda^{2} v_{2}\left(\lambda^{2} t\right) .
\end{aligned}
$$

In the first and third terms we have used the preceding estimates, $\Psi^{\prime}\left(\lambda^{2} t\right) \leqq 1$, and the bound $\left|\left(\frac{F^{\prime \prime}}{F^{\prime}}\right)^{\prime}\right| \leqq L[$ see (99)]. In the second term we have used the definition of $\|\delta F\|_{2}$; note moreover that, by the concavity of $\Psi, \Psi^{\prime}\left(\lambda^{2} t\right) \leqq\left(\lambda^{2} t\right)^{-1} \Psi\left(\lambda^{2} t\right)$ so the second term is majorized by

$$
\lambda^{2}\left(\lambda^{2} t\right)^{-1} \Psi\left(\lambda^{2} t\right)\left(1-\Psi\left(\lambda^{2} t\right)\right)\|\delta F\|_{2} .
$$


Since $y \rightarrow y(1-y)$ is increasing for $0 \leqq y<\frac{1}{2}, \Psi\left(\lambda^{2} t\right) \leqq \lambda^{2} t \leqq A<0.5$, this is majorized by $\lambda^{2}\left(1-\lambda^{2} t\right)\|\delta F\|_{2}$. Thus

$$
\begin{aligned}
v_{2}(t) \leqq & \left\{\frac{N \lambda^{6} t}{1-\lambda^{2}}\left[1-\frac{\lambda^{4} t}{2\left(1-\lambda^{4}\right)}\left(1-\lambda^{2}-\lambda^{2} N\left(1-\frac{\lambda^{2} A}{3\left(1+\lambda^{2}\right)}\right)\right)\right]\right. \\
& \left.+\lambda^{2}\left(1-\lambda^{2} t\right)+\frac{\lambda^{8} t^{2} L}{2\left(1-\lambda^{4}\right)}\left(1+\lambda^{2}-\frac{\lambda^{4} t}{3}\right)\right\}\|\delta F\|_{2} \\
& +\lambda^{2} v_{2}\left(\lambda^{2} t\right) .
\end{aligned}
$$

Iterating this again yields a convergent series, and, finally,

$$
\begin{aligned}
\|\delta F\|_{2}^{-1} v_{2}(t) \leqq & \frac{N \lambda^{6}}{1-\lambda^{2}}\left[\frac{t}{1-\lambda^{4}}-\frac{\lambda^{4} t^{2}}{2\left(1-\lambda^{4}\right)\left(1-\lambda^{6}\right)}\right. \\
& \left.\cdot\left(1-\lambda^{2}-\lambda^{2} N\left(1-\frac{\lambda^{2} A}{3\left(1+\lambda^{2}\right)}\right)\right)\right] \\
& +\frac{\lambda^{2}}{1-\lambda^{4}}\left(1+\lambda^{2}-\lambda^{2} t\right)+\frac{\lambda^{8} L t^{2}}{2\left(1-\lambda^{4}\right)}\left[\frac{1+\lambda^{2}}{1-\lambda^{6}}-\frac{\lambda^{4} t}{3\left(1-\lambda^{8}\right)}\right] .
\end{aligned}
$$

Thus:

$$
\left|\delta\left(\frac{\Psi^{\prime \prime}(t)}{\Psi^{\prime}(t)}\right)\right| \leqq\left\{\frac{\lambda^{2}}{2 A} h_{4}+\frac{\lambda^{2}}{1-\lambda^{4}}\left(1+\lambda^{2}-\lambda^{2} t\right)\right\}\|\delta F\|_{2},
$$

with

$$
\begin{aligned}
h_{4}= & \frac{A^{2} N \lambda^{2}}{\left(1-\lambda^{2}\right)\left(1-\lambda^{4}\right)\left(1-\lambda^{6}\right)}\left[2\left(1-\lambda^{6}\right)-\lambda^{2} A\left(1-\lambda^{2}-\lambda^{2} N\left(1-\frac{\lambda^{2} A}{3\left(1+\lambda^{2}\right)}\right)\right)\right] \\
& +\frac{\lambda^{2} A^{3} L}{\left(1-\lambda^{4}\right)\left(1-\lambda^{6}\right)}\left[1+\lambda^{2}-\frac{\lambda^{2} A\left(1-\lambda^{6}\right)}{3\left(1-\lambda^{8}\right)}\right] .
\end{aligned}
$$

To summarize: we have defined a linear operator $\delta F \rightarrow \delta \Psi$ which is continuous in the $\mathscr{C}^{2}$ topology. We now intend to verify that this operator is the Fréchet derivative of the map $F \rightarrow \Psi$ considered as a map of (a subset of) $\mathscr{C}^{3}$ ([0,A]) to $\mathscr{C}^{2}\left(\left[0, A \lambda^{-2}\right]\right)$. Let

$$
\begin{aligned}
& W_{3}=\left\{F \in \mathscr{C}^{3}([0, A]): F(0)=0, F^{\prime}(0)=\lambda^{-2}, F^{\prime \prime}(x) \leqq 0\right\} \\
& W_{2}=\left\{\Psi \in \mathscr{C}^{2}\left(\left[0, \frac{A}{\lambda^{2}}\right]\right): \Psi(0)=0, \Psi^{\prime}(0)=1, \Psi^{\prime \prime}(x) \leqq 0\right\}
\end{aligned}
$$

and define $H: W_{3} \times W_{2} \rightarrow W_{2}$ by

$$
H(F, \Psi)(t)=F\left(\Psi\left(\lambda^{2} t\right)\right) .
$$


Then the map $F \rightarrow \Psi$ is defined by the equation

$$
\Psi=H(F, \Psi) .
$$

Since the implicit function theorem applies to maps of Banach spaces (see e.g. [6]), it is sufficient to examine the Fréchet differentiability of $H$. We shall only indicate the principle of this verification and replace the map $(F, \Psi) \rightarrow H$ by the $\operatorname{map}\left(\frac{F^{\prime \prime}}{F^{\prime}}, \frac{\Psi^{\prime \prime}}{\Psi^{\prime}}\right) \rightarrow \frac{H^{\prime \prime}}{H^{\prime}}$ : namely

$$
\lambda^{-2} \frac{H^{\prime \prime}(t)}{H^{\prime}(t)}=\frac{F^{\prime \prime}\left(\Psi\left(\lambda^{2} t\right)\right)}{F^{\prime}\left(\Psi\left(\lambda^{2} t\right)\right)} \Psi^{\prime}\left(\lambda^{2} t\right)+\frac{\Psi^{\prime \prime}\left(\lambda^{2} t\right)}{\Psi^{\prime}\left(\lambda^{2} t\right)} .
$$

Here $\Psi^{\prime}\left(\lambda^{2} t\right)$ is interpreted as $\exp \int_{0}^{\lambda^{2} t} \frac{\Psi^{\prime \prime}(y)}{\Psi^{\prime}(y)} d y, \Psi\left(\lambda^{2} t\right)=\int_{0}^{\lambda^{2} t} \Psi^{\prime}(y) d y$. Calling $\delta\left(\frac{F^{\prime \prime}}{F^{\prime}}\right)$ and $\delta\left(\frac{\Psi^{\prime \prime}}{\Psi^{\prime}}\right)$ the increments of $\frac{F^{\prime \prime}}{F^{\prime}}$ and $\frac{\Psi^{\prime \prime}}{\Psi^{\prime}}$ respectively, one sets

$$
\delta \Psi^{\prime}(t)=\Psi^{\prime}(t) \int_{0}^{t} \delta\left(\frac{\Psi^{\prime \prime}(s)}{\Psi^{\prime}(s)}\right) d s, \quad \delta \Psi(t)=\int_{0}^{t} \delta \Psi^{\prime}(s) d s .
$$

We now have to prove that

$$
\begin{aligned}
&\left\|\delta\left(\frac{\Psi^{\prime \prime}}{\Psi^{\prime}}\right)\right\|_{\mathscr{C}_{0} 0}^{\lim }+\left\|\delta\left(\frac{F^{\prime \prime}}{F^{\prime}}\right)\right\|_{\mathscr{C}^{1}} \rightarrow 0 \sup _{t}\left[\left\|\delta\left(\frac{\Psi^{\prime \prime}}{\Psi^{\prime}}\right)\right\|+\left\|\delta\left(\frac{F^{\prime \prime}}{F^{\prime}}\right)\right\|\right]^{-1} \\
& \cdot\left(\frac{F^{\prime \prime}}{F^{\prime}}(\Psi+\delta \Psi)-\frac{F^{\prime \prime}}{F^{\prime}}(\Psi)-\left(\frac{F^{\prime \prime}}{F^{\prime}}\right)^{\prime} \delta \Psi\right) \Psi^{\prime} \\
&+\left[\frac{F^{\prime \prime}}{F^{\prime}}(\Psi+\delta \Psi)-\frac{F^{\prime \prime}}{F^{\prime}}(\Psi)\right] \cdot \delta \Psi^{\prime}+\left[\delta\left(\frac{F^{\prime \prime}}{F^{\prime}}\right)(\Psi+\delta \Psi)-\delta\left(\frac{F^{\prime \prime}}{F^{\prime}}\right)(\Psi)\right] \Psi \Psi^{\prime} \mid=0
\end{aligned}
$$

This is straightforward; the last term is the only one which requires some attention since one has to use the $\mathscr{C}^{1}$ nature of $\delta\left(\frac{F^{\prime \prime}}{F^{\prime}}\right)$, i.e. this term is bounded by $\left\|\delta\left(\frac{F^{\prime \prime}}{F^{\prime}}\right)\right\|_{\mathscr{C}^{1}}\|\delta \Psi\|_{\mathscr{C}^{0}}$

From the Fréchet differentiability of the map $F \rightarrow \Psi$ it follows that also the map $\bar{T}_{\lambda}: \mathscr{H}_{\lambda}^{\prime 1} \rightarrow \mathscr{H}_{\lambda}^{\prime 0}$ is Fréchet differentiable. The superscripts mean that we think of $\bar{T}_{\lambda}$ as a map from $\mathscr{H}_{\lambda}^{\prime}$ embedded in the Banach space $\mathscr{C}^{1}([0, A])$ into $\mathscr{H}_{\lambda}^{\prime}$ embedded in the Banach space $\mathscr{C}([0, A])$.

We are now in a position to estimate the derivative of the map $F \rightarrow G$ or, equivalently, $D \bar{T}_{\lambda}$. We use the formulae

$$
\begin{aligned}
\frac{1}{(1-x)} \delta\left(\frac{G^{\prime \prime}(x)}{G^{\prime}(x)}\right)= & \left\{\delta \alpha\left[-2 \frac{\Psi^{\prime \prime}(t)}{\Psi^{\prime}(t)}-2 \alpha(1-x)^{2} \sigma(t)\right]\right. \\
& \left.-2 \alpha \delta\left(\frac{\Psi^{\prime \prime}(t)}{\Psi^{\prime}(t)}\right)\right\}_{t=\alpha(1-x)^{2}},
\end{aligned}
$$


where $0 \leqq t \leqq A \lambda^{-2}$ and $\sigma(t) \equiv \frac{d}{d t}\left(\frac{\Psi^{\prime \prime}(t)}{\Psi^{\prime}(t)}\right)$. Using the estimates (55) and (95) found for $\left|\Psi^{\prime \prime}(t) / \Psi^{\prime}(t)\right|$ and $|\sigma(t)|$, and the preceding estimates for $\delta \alpha$ and $\delta\left(\Psi^{\prime \prime}(t) / \Psi^{\prime}(t)\right)$ we obtain

$$
\begin{aligned}
\left|\frac{1}{1-x} \delta\left(\frac{G^{\prime \prime}(x)}{G^{\prime}(x)}\right)\right| \leqq & \left\{h_{3}\left[2 \beta_{0} \frac{A}{\lambda^{2}}+2 \beta_{1} \frac{A t}{\lambda^{2}}+\frac{2 t A \lambda^{2} L}{1-\lambda^{4}}\right]\right. \\
& \left.+\frac{\alpha \lambda^{2}}{A} h_{4}+\frac{2 \alpha \lambda^{2}}{1-\lambda^{4}}\left(1+\lambda^{2}-\lambda^{2} t\right)\right\}\|\delta F\|_{2} .
\end{aligned}
$$

Denoting $t=\frac{A}{\lambda^{2}} \Theta$, so that $\Theta=\frac{\lambda^{2} \alpha}{A}(1-x)^{2}$, we thus obtain (taking into account that $\alpha \leqq A \lambda^{-2}$ ) the upper bound :

$$
\left\{h_{3}\left[\ell_{1}+\Theta\left(\ell_{3}+\frac{2 A^{2} L}{1-\lambda^{4}}\right)\right]+h_{4}+\frac{2 A}{1-\lambda^{4}}\left(1+\lambda^{2}-A \Theta\right)\right\}\|\delta F\|_{2} .
$$

This is a linear function of $\Theta$ which is increasing if

$$
h_{3}\left[\left(1-\lambda^{4}\right) \ell_{3}+2 A^{2} L\right]-2 A^{2} \geqq 0 .
$$

In this case (the only one we shall actually encounter) the maximum of this linear function is its value at $\Theta=\frac{\alpha^{2} \lambda}{A} \leqq 1$ so that the norm of $D \bar{T}_{\lambda}$ is bounded by

$$
\left\|D \bar{T}_{\lambda}\right\| \leqq h_{3}\left[\ell_{1}+\ell_{3}+\frac{2 A^{2} L}{1-\lambda^{4}}\right]+h_{4}+\frac{2 A}{1-\lambda^{4}}\left(1+\lambda^{2}-A\right) .
$$

In case the expression (157) is negative, an upper bound for $\left\|D \bar{T}_{\lambda}\right\|$ is obtained by inserting in (156) the value $\Theta=\frac{\alpha_{\min } \lambda^{2}}{A}(1-A)^{2}$ or (worse) $\frac{\lambda}{2 A}(1-A)^{2}$.

Interval Calculations. Let $J$ be an interval $\left\{\lambda: p \leqq \lambda^{2} \leqq b\right\}$. We denote $\mathscr{H}_{\boldsymbol{J}}^{\prime}\left(\ell_{1}, \ell_{3}, c_{1}, c_{3}, A\right)$ the subset of $\mathscr{C}^{1}([0, A])$ defined by

$\left\{s \in \mathscr{C}^{1}([0, A]): s^{\prime}(x)+s(x)^{2} \leqq 0,-s^{\prime}(x) \leqq L_{J}\right.$,

$\left.\frac{1}{1-x}-\ell_{1}(1-x)-\ell_{3}(1-x)^{3} \leqq-s(x) \leqq \frac{1}{1-x}-c_{1}(1-x)-c_{3}(1-x)^{3}\right\}$.

Here $L_{J}$ is obtained by substituting $b$ for $\lambda^{2}$ in (99). The set (159) is identical to $k^{-1} \mathscr{S}_{J}\left(\ell_{1}, \ell_{3}, c_{1}, c_{3}, A\right)$. An examination of the bounds just obtained for the norm of $D \bar{T}_{\lambda}(s)$ at $s \in \mathscr{H}_{\lambda}^{\prime}$ shows that these estimates are increasing functions of $\lambda^{2}$. Hence for $\lambda \in J$, for $s \in \mathscr{H}_{J}^{\prime}\left(\ell_{1}, \ell_{3}, c_{1}, c_{3}, A\right)$, a bound of $\left\|D \bar{T}_{\lambda}(s)\right\|$ is obtained by replacing $\lambda^{2}$ with $b$ in these estimates.

\section{Numerical Results.}

This section contains the numerical results obtained by applying the various estimates of the preceding sections. Table 1 provides, for certain values of $\lambda^{2}$, 
values of $c_{1}, c_{3}, \ell_{1}, \ell_{3}$ and $A$ such that $\mathscr{S}_{\lambda}\left(\ell_{1}, \ell_{3}, c_{1}, c_{3}, A\right)$ is stable [i.e. Criterion 1 is satisfied and $\left.\ell_{j}^{\prime}\left(c_{1}, c_{3}, A, \lambda^{2}\right)<\ell_{j}, j=1,3, c_{j}^{\prime}\left(\ell_{1}, \ell_{3}, c_{1}, c_{3}, A, \lambda\right)>c_{j}, j=1,3\right]$. Only the first and last rows in this table are indispensable for our proof, the others being supplied for completeness. The eleventh column contains an upper bound for the norm of $D \bar{T}_{\lambda}$ evaluated with the formulae of the preceding section.

Furthermore by applying procedure 3 (with 4 iterations), to the last case $\left(\lambda^{2}=0.165\right)$ one finds that at any fixed point corresponding to those values,

$$
\alpha<\alpha_{f}=1.5334, \quad A_{f}=\lambda^{2} \alpha_{f}=0.253011
$$

and consequently such a fixed point verifies (for $0 \leqq x \leqq A_{f}$ ) [see (103)]

$$
\begin{aligned}
M_{f}=\sup _{0 \leqq t \leqq A_{f} \lambda^{-2}} \frac{\Psi_{-}^{\prime \prime \prime}(t)}{\Psi^{\prime}(t)} & \leqq \frac{3 \lambda^{4} A_{f}}{A}\left(\ell_{1}+\ell_{3}\right)\left(1-\lambda^{4}-4 A_{f}^{2}\right)^{-1} \\
& \leqq 0.07398
\end{aligned}
$$

Table 2 presents the same data for a selection of intervals.

Existence of Fixed Points. Table 2 shows that, restricted to the corresponding $\mathscr{H}_{J}^{\prime}$, the map $\bar{T}_{\lambda}$ has a derivative $D \bar{T}_{\lambda}$ such that its $\mathscr{C}^{0} \rightarrow \mathscr{C}^{0}$ norm in the sense of (135) is $\leqq \kappa<1$ (i.e. $\left.\|\delta G\|_{2} \leqq \kappa\|\delta F\|_{2}\right)$. Let $s_{1}, s_{2} \in \mathscr{H}_{J}^{\prime}\left(\ell_{1}, \ell_{3}, c_{1}, c_{3}, A\right)$ for one of the sets of constants appearing in Table 2 . Then, for $\lambda \in J$,

$$
\bar{T}_{\lambda}\left(s_{2}\right)-\bar{T}_{\lambda}\left(s_{1}\right)=\int_{0}^{1} d y \frac{d}{d y} \bar{T}_{\lambda}\left(y s_{2}+(1-y) s_{1}\right) .
$$

Since $\mathscr{H}_{J}^{\prime}$ is a convex set, $\bar{T}_{\lambda}\left(y s_{2}+(1-y) s_{1}\right)$ is a continuously differentiable vector valued function of $y$ and

$$
\frac{d}{d y} \bar{T}_{\lambda}\left(y s_{2}+(1-y) s_{1}\right)=D \bar{T}_{\lambda}\left(y s_{2}+(1-y) s_{1}\right) \cdot\left(s_{2}-s_{1}\right)
$$

so that

$$
\left\|\bar{T}_{\lambda}\left(s_{1}\right)-\bar{T}_{\lambda}\left(s_{2}\right)\right\| \leqq \kappa\left\|s_{2}-s_{1}\right\|
$$

[The norm being as defined by (135)]. Since it has already been seen that $\bar{T}_{\lambda}$ is continuous in the $\mathscr{C}^{0}$ topology (because $T_{\lambda}$ is continuous in the $\mathscr{C}^{2}$ topology) the inequality (161) immediately extends to the closure $\overline{\mathscr{H}}_{J}^{\prime}\left(\ell_{1}, \ell_{3}, c_{1}, c_{3}, A\right)$ of $\mathscr{H}_{J}^{\prime}$ in the norm \|\| (this space consists of Lipschitzian continuous functions). Thus, for every $\lambda \in J, \bar{T}_{\lambda}$ has an unique fixed point in $\overline{\mathscr{H}}_{J}^{\prime}$. Moreover since the intersection of $\overline{\mathscr{H}}_{J}^{\prime}$ with $\left\{\frac{F^{\prime \prime}}{F^{\prime}}: F \in \mathscr{A}_{B}\right\}$ is non empty, closed, and stable under $\bar{T}_{\lambda}$, the fixed point is contained in this set. Finally $\bar{T}_{\lambda}$ depends continuously on $\lambda$ in the $\mathscr{C}^{0}$ topology, so that the fixed point is a continious function of $\lambda$ in this topology. Note that if $\lambda \in J_{1} \cap J_{2}$ (as is the case e.g. for $\lambda^{2}=0.161, \lambda^{2}=0.164$ ) and if (this is the case for the values of the above table, as demonstrated by the example in Sect.8) $\overline{\mathscr{H}}_{J_{1}}^{\prime} \cap \overline{\mathscr{H}}_{J_{2}}^{\prime} \neq \emptyset$, this set, being stable under $\bar{T}_{\lambda}$, contains the unique fixed point corresponding to both $J_{1}$ and $J_{2}$, i.e. the fixed point is a continuous function of $\lambda$ in $J_{1} \cup J_{2}$. 


\section{Conclusion}

Theorem 3. For all $\lambda$ in the interval $0.152 \leqq \lambda^{2} \leqq 0.165, \bar{T}_{\lambda}$ has a unique fixed point $s_{\lambda}$ such that $s_{\lambda} \in \overline{\mathscr{H}}_{J_{j}}^{\prime}$ when $\lambda \in J_{j}, J_{1}, J_{2}, J_{3}$ being the intervals described by Table 2 and $\overline{\mathscr{H}}_{J_{J}}^{\prime}$ being defined with the constants given there. This fixed point continuously depends on $\lambda$ and $k^{-1} s_{\lambda} \in \mathscr{A}_{B}$ for all $B \geqq 1.8$.

Let $F_{\lambda}$ denote $k^{-1} s_{\lambda}$, i.e.

$$
\left\{\begin{array}{l}
F_{\lambda}^{\prime}(x)=\lambda^{-2} \exp \int_{0}^{x} s_{\lambda}(y) d y, \quad 0 \leqq x \leqq A \\
F_{\lambda}(x)=\int_{0}^{x} F_{\lambda}^{\prime}(y) d y .
\end{array}\right.
$$

Then $G_{\lambda}=T_{\lambda} F_{\lambda}$ coincides with $F_{\lambda}$ for $0 \leqq x \leqq A$, and we define $F_{\lambda}(x)=G_{\lambda}(x)$ for $0 \leqq x \leqq 1$. Note that $G_{\lambda} \in \mathscr{A}_{B}$ since $F_{\lambda} \mid[0, A] \in \mathscr{A}_{B}$, according to the estimates of Sect. 7 .

We also denote $\chi_{\lambda}$ the function defined on $[-1,1]$ by

$$
\chi_{\lambda}(x)=\lambda\left[F_{\lambda}(1-x)-F_{\lambda}(1)\right]+1 \quad \text { for } \quad 0 \leqq x \leqq 1, \quad \chi_{\lambda}(x)=\chi_{\lambda}(-x)
$$

It also satisfies

$$
\chi_{\lambda}(x)=1-\psi_{\lambda}\left(x^{2}\right)=1-\Psi_{\lambda}\left(\alpha x^{2}\right)
$$

with obvious notations.

\section{Existence of Feigenbaum's Fixed Point}

We shall now complete the proof of this fact by proving that the continuous function of $\lambda$ defined by $\chi_{\lambda}(1)+1$ takes values of opposite signs at $\lambda^{2}=0.152$ and $\lambda^{2}=0.165$.

We have

$$
\begin{aligned}
\chi_{\lambda}(1)+\lambda & =1+\lambda-\Psi_{\lambda}(\alpha), \\
\Psi_{\lambda}(\alpha) & =\int_{0}^{\alpha} \Psi_{\lambda}^{\prime}(t) d t .
\end{aligned}
$$

We know that $\Psi_{\lambda}^{\prime}(t)$ decreases from 1 to $\Psi_{\lambda}^{\prime}(\alpha)=(2 \lambda \alpha)^{-1}$ as $t$ increases from 0 to $\alpha$. Thus

$$
\Psi_{\lambda}^{\prime}(t)=1-t\left(\frac{1-\Psi_{\lambda}^{\prime}(\alpha)}{\alpha}\right)+f_{\lambda}(t),
$$

where $f_{\lambda}(0)=f(\alpha)=0, f_{\lambda}^{\prime \prime}(t)=\Psi_{\lambda}^{\prime \prime \prime}(t)$, and :

$$
\Psi_{\lambda}(\alpha)=\int_{0}^{\alpha} f_{\lambda}(t) d t+\frac{\alpha}{2}+\frac{1}{4 \lambda}
$$


Moreover

$$
f_{\lambda}(t)=\int_{0}^{t}(t-y) f_{\lambda}^{\prime \prime}(y) d y+\kappa t
$$

where $\kappa$ is determined by $f_{\lambda}(\alpha)=0$, i.e.

$$
\begin{aligned}
& f_{\lambda}(t)=\int_{0}^{t}(t-y) f_{\lambda}^{\prime \prime}(y) d y-\frac{t}{\alpha} \int_{0}^{\alpha}(\alpha-y) f_{\lambda}^{\prime \prime}(y) d y, \\
& f_{\lambda}(t)=-\int_{0}^{t} t\left(\frac{y}{t}-\frac{y}{\alpha}\right) f_{\lambda}^{\prime \prime}(y) d y-\frac{t}{\alpha} \int_{t}^{\alpha}(\alpha-y) f_{\lambda}^{\prime \prime}(y) d y .
\end{aligned}
$$

Here $0 \leqq y \leqq t \leqq \alpha$ so that $\frac{y}{t}-\frac{y}{\alpha} \geqq 0, \alpha-y \geqq 0$. As a consequence,

$$
f_{\lambda}(t) \leqq \int_{0}^{t} t\left(\frac{y}{t}-\frac{y}{\alpha}\right) f_{\lambda}^{\prime \prime}-(y)+\frac{t}{\alpha} \int_{0}^{\alpha}(\alpha-y) f_{\lambda-}^{\prime \prime}(y) d y
$$

In particular we know that $f_{\lambda-}^{\prime \prime}(y)=\Psi_{\lambda^{-}}^{\prime \prime \prime}(y) \leqq \frac{\Psi_{\lambda}^{\prime \prime \prime}-(y)}{\Psi_{\lambda}^{\prime}(y)} \leqq M_{f}$ where $M_{f}$ is evaluated in (160). Hence

$$
f_{\lambda}(t) \leqq \frac{M_{f}}{2} t(\alpha-t)
$$

and

$$
\int_{0}^{\alpha} f_{\lambda}(t) d t \leqq \frac{M_{f} \alpha^{3}}{12} \leqq \frac{M_{f} \alpha_{f}^{3}}{12}
$$

For $\lambda^{2}=0.165$ we find

$$
\chi_{\lambda}(1)+\lambda \geqq 1+\lambda-\frac{\alpha_{f}}{2}-\frac{1}{4 \lambda}-\frac{M_{f} \alpha_{f}^{3}}{12} \geqq 0.0018 .
$$

On the other hand, taking $\lambda^{2}=0.152$ and using again (164), we have

$$
\begin{aligned}
f_{\lambda}(t) & \geqq-\int_{0}^{t} t y\left(\frac{1}{t}-\frac{1}{\alpha}\right) f_{\lambda^{+}}^{\prime \prime}(y) d y-\frac{t}{\alpha} \int_{t}^{\alpha}(\alpha-y) f_{\lambda^{+}}^{\prime \prime}(y) d y \\
& \geqq-\frac{m \alpha^{3}}{12}, \quad m=\sup _{t} f_{\lambda^{+}}^{\prime \prime}(t)=\sup _{t} \Psi_{\lambda^{+}}^{\prime \prime}(t) \leqq \sup _{t} \frac{\Psi_{\lambda^{+}}^{\prime \prime}(t)}{\Psi_{\lambda^{\prime}}^{\prime}(t)} .
\end{aligned}
$$

This has been estimated in formula (126). Using the figures in Table 1, we find, for $\lambda=0.152$,

$$
\begin{aligned}
m & \leqq 0.01374 \\
\chi_{\lambda}(1)+\lambda & \leqq 1+\lambda-\frac{\alpha}{2}-\frac{1}{4 \lambda}+\frac{m \alpha^{3}}{12} \\
& \leqq 1+\lambda-\frac{\alpha_{\min }}{2}\left(1-\frac{m \alpha_{\min }^{2}}{6}\right)-\frac{1}{4 \lambda} \\
& \leqq-0.0444
\end{aligned}
$$


This proves:

Theorem 4. There is (at least one) value $\lambda_{0}$ of $\lambda$ in the interval $0.152 \leqq \lambda^{2} \leqq 0.165$ such that $\chi_{\lambda_{0}}(1)+\lambda_{0}=0$, and hence

$$
\begin{aligned}
& \chi_{\lambda_{0}}(x)=-\frac{1}{\lambda_{0}}\left(\chi_{\lambda_{0}}\left(\lambda_{0} x\right)\right), \\
& \chi_{\lambda_{0}}(0)=1 .
\end{aligned}
$$

This function is denoted $g$ in the sequel. It has the following properties:

1) $g$ is even, $\mathscr{C}^{\infty}$ on $[-1,1]$ and satisfies, for all $x \in[0,1]: g^{\prime \prime \prime}(x) \geqq 0, g^{\prime \prime}(x) \leqq 0$, $g^{\prime}(x) \leqq 0, g(1)=-\lambda_{0}, g^{\prime}(1)=-\lambda_{0}^{-1}, g^{\prime \prime}(0)=-2 \alpha\left(\right.$ since $\left.g(x)=1-\Psi\left(\alpha x^{2}\right)\right)$.

2) $g$ is holomorphic in a complex neighborhood of $[-1,1]$. For all $n \geqq 1$, $x \in[-1,1]$

$$
\left|\frac{1}{n !}\left(\frac{d}{d x}\right)^{n} g(x)\right| \leqq \frac{1}{\lambda_{0}} B_{0}^{n-1}, \quad B_{0}=1.8
$$

and $g(x)=1-\Psi\left(\alpha x^{2}\right), \Psi(0)=0, \Psi^{\prime}(0)=1$, and for $0 \leqq t \leqq \lambda_{0}^{-2}$,

$$
\left|\frac{1}{n !}\left(\frac{d}{d t}\right)^{n} \Psi(t)\right| \leqq M_{0}^{n-1}, \quad M_{0}=\lambda_{0}^{2}\left(1-\lambda_{0}^{2}\right)^{-1} B_{0} .
$$

The numbers $\lambda_{0}$ and $\alpha$ verify

$$
0.152 \leqq \lambda_{0}^{2}<0.165, \quad 1.429<\alpha<1.615 .
$$

Moreover $\left(M_{0} \alpha\right)^{-1}>1.79$ so that $g$ is, in particular, holomorphic in the disk $\left|x^{2}\right|<1.79$.

\section{Additional Remarks}

1. Further Elementary Properties of $g$. It has been seen that $g$ is analytic in a certain neighborhood of $[-1,1]$ in $\mathbb{C}$. If any subset $\Omega=-\Omega$ of this neighborhood has the property that $g(\Omega) \subset \Omega$ then $g$ can be extended to $\frac{1}{\lambda_{0}} \Omega$ by using (1), and is analytic in a neighborhood of this new set; moreover $g\left(\frac{1}{\lambda_{0}} \Omega\right) \subset-\frac{1}{\lambda_{0}} g(\Omega) \subset \frac{1}{\lambda_{0}} \Omega$ so that the process can be iterated indefinitely. In particular taking $\Omega=[-1,1]$ shows that $g$ is defined and analytic on the whole real axis. Since $\Psi(t)$ is analytic and positive for $0 \leqq-t \leqq k^{2}$, taking $\Omega=\mathbb{R} \cup i[-k, k]$ shows $g$ is analytic and real on the whole imaginary axis.

Note that $(S g)(x) \equiv g^{\prime \prime \prime}(x) g^{\prime}(x)^{-1}-\frac{3}{2}\left(g^{\prime \prime}(x) / g^{\prime}(x)\right)^{2} \leqq 0$ on $[-1,1]$. Since for any two functions $f$ and $h, S(f \circ h)(x)=h^{\prime}(x)^{2} S f(h(x))+S h(x), S(f)=S(-f)$, we see that $(S g)(x) \leqq 0$ for all $x \in \mathbb{R}$.

2. The Case $\varepsilon \neq 1$. The method of this paper can at least be formulated for $\varepsilon \neq 1$ (see Introduction). One then wishes to find a fixed point of the mapping $\phi_{1} \rightarrow \phi_{2}$ 
defined by Eq. (11) in the case when

$$
\phi_{2}(x)=1-\psi\left(|x|^{1+\varepsilon}\right), \quad \psi^{\prime}(0) \neq 0,
$$

$\psi(t)$ being $\mathscr{C}^{2}$ in $[0,1]$. Defining $F$ and $G$ by (14) and (15) we see that the following equations must be fulfilled:

$$
\begin{aligned}
F(0) & =0, \\
\psi(t) & =F\left(\psi\left(\lambda^{1+\varepsilon} t\right)\right), \quad \psi(0)=0, \\
G(x) & =\frac{1}{\lambda}\left[\psi(1)-\psi\left(|1-x|^{1+\varepsilon}\right)\right] .
\end{aligned}
$$

We require $F$ to be $\mathscr{C}^{2}$ for $0 \leqq x<1$. Differentiating (167) gives

$$
\psi^{\prime}(0)=\lambda^{1+\varepsilon} \psi^{\prime}(0) F^{\prime}(0)
$$

and the condition $\psi^{\prime}(0) \neq 0$ imposes $F^{\prime}(0)=\lambda^{-(1+\varepsilon)}$. For this to be verified also by $G, \psi$ must satisfy

$$
\psi^{\prime}(1)=\left[(1+\varepsilon) \lambda^{\varepsilon}\right]^{-1}
$$

We may again look for $\psi$ in the form $\psi(t)=\Psi(\alpha t), \Psi^{\prime}(0)=1, \alpha$ being determined by: $\alpha \Psi^{\prime}(\alpha)=\left[(1+\varepsilon) \lambda^{\varepsilon}\right]^{-1}$. The functional equation $\Psi(t)=F\left(\Psi\left(\lambda^{1+\varepsilon} t\right)\right), \quad \Psi(0)=0$, $\Psi^{\prime}(0)=1$ is solved as in Lemma 1 .

There seems to be no reason for the rest of the method not to work, at least for $\varepsilon$ close to 1 , and possibly also for $0<\varepsilon \leqq 1$.

3. We note also that the method can be reformulated to attack directly the mapping $J$ [see Eq. (5)]. However estimates are more difficult and have not, so far, been carried out to the end. This method would have the advantage of leading to uniqueness (at least locally) of $\lambda_{0}$ and $g$. Note that this has not been demonstrated here but that, for every solution $\lambda_{0}$ of the equation $\chi_{\lambda}(1)+\lambda=0$, the corresponding $g=\chi_{\lambda_{0}}$ is locally unique, since it is given by a contraction.

4. Martin has studied conditions under which a solution $(\varepsilon=1)$ could exist with a very different shape from the concave one obtained here: it turns out that this would require some rather violent oscillations (private communication).

\section{Some Properties of the Linearized Map at the Fixed Point}

Feigenbaum's theory relies on the spectral properties of the derivative of the map $J$ at the fixed point (see $[2,3]$ ). For this purpose it is equivalent to consider the derivative of the map $m(\cdot, \lambda)$ with the $\lambda$ corresponding to the fixed point, since the two operators are very simply related. We state without proof some results which follow straightforwardly from the proven properties of the fixed point $g$.

We consider the derivative of $m(\cdot, \lambda)$ :

$$
A u(x)=-\frac{1}{\lambda}\left[u(g(\lambda x))+g^{\prime}(g(\lambda x)) u(-\lambda x)\right] .
$$


Given a real $R>0$, we denote by $\Delta_{R}$ the domain:

$$
\Delta_{R} \equiv\{r \in \mathbb{C} \mid r=x+u \text {, with } x \in[-1,1] \text { and }|u|<R\} .
$$

We consider the Banach space $H\left(\Delta_{R}\right)$ of the analytic functions $u$ in $\Delta_{R}$, such that $u(x)$ is real for real $x$ and $u^{\prime}(0)=0$, with the norm of the sup. We define also the Banach space of the even functions in $H\left(\Delta_{R}\right)$ :

$$
H^{+}\left(\Delta_{R}\right)=\left\{u \in H\left(\Delta_{R}\right) \mid u(r)=u(-r) \forall r \in \Delta_{R}\right\}
$$

and the closed cones:

$$
\begin{aligned}
& K_{R}^{+} \equiv\left\{u \in H^{+}\left(\Delta_{R}\right) \mid \forall x \in[0,1], u(x) \geqq 0, u^{\prime}(x) \leqq 0\right\}, \\
& K_{R}=\left\{u \in H\left(\Delta_{R}\right) \mid \forall x \in[-1,1], \quad u(x) \geqq 0 \geqq x u^{\prime}(x) ;\right.
\end{aligned}
$$

for all integer $n \geqq 0$,

$$
\left.\min \left[u\left(\lambda^{n+1}\right), u\left(-\lambda^{n+1}\right)\right] \geqq \max \left[u\left(\lambda^{n} g(\lambda)\right), u\left(-\lambda^{n} g(\lambda)\right)\right]\right\} \text {. }
$$

We have $K_{R}^{+}=K_{R} \cap H^{+}\left(\Delta_{R}\right)$. We denote $K_{R}^{0}$ (respectively $K_{R}^{+0}$ ) the interior of $K_{R}$ (respectively $K_{R}^{+}$) in $H\left(\Delta_{R}\right)$ [respectively $H^{+}\left(\Delta_{R}\right)$ ]. If $R_{2}>R_{1}>0$, there is a natural compact embedding of $H\left(\Delta_{R_{2}}\right)$ into $H\left(\Delta_{R_{1}}\right)$. The following propositions hold:

Proposition 1. There exist $R_{1}>0$ and $R_{2}>0$ such that $A$ is a bounded linear operator from $H\left(\Delta_{R_{1}}\right)$ into $H\left(\Delta_{R_{2}}\right)$. As an operator from $H\left(\Delta_{R_{1}}\right)$ into itself, $A$ is compact.

Proposition 2. The following inclusions are verified

(i) $A\left(K_{R_{1}} \backslash\{0\}\right) \subset K_{R_{1}}^{0}$,

(ii) $A\left(K_{R_{1}}^{+} \backslash\{0\}\right) \subset K_{R_{1}}^{+0}$.

Using Propositions 1 and 2 and Theorem 6.3 of Krein and Rutman [10], we obtain

Theorem. (i) $A$ has one and only one eigenvector $v$ in $K_{R_{1}}$. This vector belongs to $K_{R_{1}}^{+0}$.

(ii) The corresponding eigenvalue $\varrho$ verifies $\varrho \geqq \lambda^{-2}-\lambda^{-1}$.

(iii) The adjoint operator $A^{*}$ has, in $K_{R_{1}}^{+}$, one and only one eigenvector $\psi$ : this is a strictly positive functional verifying $A^{*} \psi=\varrho \psi$.

We note that the inequality for $\varrho$ follows from the fact that, for $u \in K_{R_{1}}^{+}$, we have

$$
A u(0)=-\lambda^{-1}\left[u(1)+g^{\prime}(1) u(0)\right]=\lambda^{-2} u(0)-\lambda^{-1} u(1) \geqq\left(\lambda^{-2}-\lambda^{-1}\right) u(0) .
$$

Sketch of Proof of Proposition 2 (i). Denote $b=g(\lambda)=1-\Psi\left(\lambda^{2} \alpha\right) \geqq 1-A>\lambda$, and $h(x)=-g^{\prime}(g(\lambda x))$ : this is a positive, even function on $[-1,1]$ and decreasing on $[0,1]$. Since $h(x)=\lambda F^{\prime}\left(\Psi\left(\alpha \lambda^{2} x^{2}\right)\right)=H(t)$ for $t=\alpha x^{2}$,

$$
-\frac{d}{d t} H(t)=-\lambda^{3} F^{\prime \prime}\left(\Psi\left(\lambda^{2} t\right)\right) \Psi^{\prime}\left(\lambda^{2} t\right) \geqq-\lambda^{3} F^{\prime \prime}(0) \Psi^{\prime}\left(\lambda^{2} \alpha\right) .
$$

Hence, for $n \geqq 0$

$$
\begin{gathered}
h\left(\lambda^{n+1}\right)-h\left(\lambda^{n} b\right) \geqq a \lambda^{2 n}, \\
a=-\alpha \lambda^{3} F^{\prime \prime}(0) \Psi^{\prime}\left(\lambda^{2} \alpha\right)\left[b^{2}-\lambda^{2}\right]>0 .
\end{gathered}
$$


Moreover $h(x) \geqq 2 \alpha b \Psi^{\prime}\left(\alpha b^{2}\right)>b \lambda^{-1} \geqq(1-A) \lambda^{-1}>1$. Now let $u \in K_{R}$. Then

$$
\begin{aligned}
\lambda A u(x) & =h(x) u(-\lambda x)-u(g(\lambda x)) \\
& \geqq b \lambda^{-1} u(-\lambda \operatorname{sgn} x)-u(b) \geqq\left(b \lambda^{-1}-1\right) u(-\lambda \operatorname{sgn} x)>0 .
\end{aligned}
$$

The last expression cannot vanish unless $u$ vanishes on an interval and hence everywhere. It is easy to check that $x A u^{\prime}(x) \leqq 0$ and (by analyticity) $x^{-1} A u^{\prime}(x)<0$. Moreover, for $n \geqq 0, \varepsilon_{1,2}= \pm 1$,

$$
\begin{aligned}
& \lambda A u\left(\varepsilon_{1} \lambda^{n+1}\right)-\lambda A u\left(\varepsilon_{2} \lambda^{n} b\right) \\
&= h\left(\lambda^{n+1}\right) u\left(-\varepsilon_{1} \lambda^{n+2}\right)-h\left(\lambda^{n} b\right) u\left(-\varepsilon_{2} \lambda^{n+1} b\right) \\
&+u\left(g\left(\lambda^{n+1} b\right)\right)-u\left(g\left(\lambda^{n+2}\right)\right) \\
& \geqq {\left[h\left(\lambda^{n+1}\right)-h\left(\lambda^{n} b\right)\right] \min \left(u\left(\lambda^{2}\right), u\left(-\lambda^{2}\right)\right) } \\
& \geqq a \lambda^{2 n} \min \left(u\left(\lambda^{2}\right), u\left(-\lambda^{2}\right)\right) .
\end{aligned}
$$

This cannot vanish unless $u=0$. Assume now that $v \in H\left(\Delta_{R}\right)$ belongs to a neighborhood $U$ of $A u$ such that

$$
\sup _{-1 \leqq x \leqq 1}\left|(A u)^{\prime \prime}(x)-v^{\prime \prime}(x)\right|<\eta .
$$

Then using $v^{\prime}(0)=(A u)^{\prime}(0)=0$,

$$
\begin{aligned}
& v\left(\varepsilon_{1} \lambda^{n+1}\right)-v\left(\varepsilon_{2} \lambda^{n} b\right)= A u\left(\varepsilon_{1} \lambda^{n+1}\right)-A u\left(\varepsilon_{2} \lambda^{n} b\right) \\
&+\int_{\varepsilon_{1} \lambda^{n+1}}^{\varepsilon_{2} \lambda^{n} b} d x \frac{d}{d x}[v(x)-A u(x)] \\
& \geqq \lambda^{2 n}\left[\frac{a}{\lambda} \min \left(u\left(\lambda^{2}\right), u\left(-\lambda^{2}\right)\right)-2 b^{2} \eta\right] .
\end{aligned}
$$

This is $>0$ for sufficiently small $\eta$, and it is easy to verify that, for sufficiently small $\eta, v(x) \geqq 0$ and $x v^{\prime}(x) \leqq 0$ for all $x \in[-1,1]$.

Acknowledgements. The authors are much indebted to J.-P. Eckmann and P. Collet without whose ecouragement and friendly advice this work could not have been carried out. One of us (M.C.) acknowledges very kind hospitality at I.H.E.S.

\section{References}

1. Campanino, M., Epstein, H., Ruelle, D.: On Feigenbaum's functional equation (to appear)

2. Collet, P., Eckmann, J.-P.: Properties of continuous maps of the interval to itself. In: Mathematical problems in theoretical physics, Proceedings, Lausanne 1979. Berlin, Heidelberg, New York: Springer 1980

3. Collet, P., Eckmann, J.-P.: Iterated maps on the interval as dynamical systems. Boston: Birkhaeuser 1980

4. Collet, P., Eckmann, J.-P., Koch, H.: Period doubling bifurcations for families of maps on $R^{n}$. Preprint, University of Geneva (1979) (to appear)

5. Collet, P., Eckmann, J.-P., Lanford, O.E., III : Commun. Math. Phys. 76, 211-254 (1980) 
6. Dieudonné, J.: Foundations of modern analysis. New York: Academic Press 1969

7. Feigenbaum, M.J.: J. Stat. Phys. 19, 25-52 (1978)

8. Feigenbaum, M.J.: J. Stat. Phys. 21, 669-706 (1979)

9. Feigenbaum, M.J.: The transition to aperiodic behavior in turbulent systems. Commun. Math. Phys. (to appear)

10. Krein, M.G.. Rutman, M.A.: Usp. Mat. Nauk 3, 1 (23), 3-95 (1948); Engl. Transl.: Functional analysis and measure theory. Am. Math. Soc., Providence 1962

11. Lanford, O.E., III: Remarks on the accumulation of period-doubling bifurcations. In: Mathematical problems in theoretical physics, Proceedings, Lausanne 1979. Berlin, Heidelberg, New York: Springer 1980.

Please note that this list contains only those references directly connected to the present paper and that they contain a more complete guide to the literature.

Communicated by D. Ruelle

Received October 13, 1980 